\title{
Schwinger effect in 4D de Sitter space and constraints on magnetogenesis in the early universe
}

\author{
Takeshi Kobayashi ${ }^{a, b}$ and Niayesh Afshordi ${ }^{b, c}$ \\ ${ }^{a}$ Canadian Institute for Theoretical Astrophysics, University of Toronto, \\ 60 St. George Street, Toronto, Ontario M5S 3H8, Canada \\ ${ }^{b}$ Perimeter Institute for Theoretical Physics, \\ 31 Caroline Street North, Waterloo, Ontario N2L 2Y5, Canada \\ ${ }^{c}$ Department of Physics and Astronomy, University of Waterloo, \\ 200 University Avenue West, Waterloo, Ontario, N2L 3G1, Canada \\ E-mail: takeshi@cita.utoronto.ca, nafshordi@pitp.ca
}

ABSTRACT: We investigate pair creation by an electric field in four-dimensional de Sitter space. The expectation value of the induced current is computed, using the method of adiabatic regularization. Under strong electric fields the behavior of the current is similar to that in flat space, while under weak electric fields the current becomes inversely proportional to the mass squared of the charged field. Thus we find that the de Sitter space obtains a large conductivity under weak electric fields in the presence of a charged field with a tiny mass. We then apply the results to constrain electromagnetic fields in the early universe. In particular, we study cosmological scenarios for generating large-scale magnetic fields during the inflationary era. Electric fields generated along with the magnetic fields can induce sufficiently large conductivity to terminate the phase of magnetogenesis. For inflationary magnetogenesis models with a modified Maxwell kinetic term, the generated magnetic fields cannot exceed $10^{-30} \mathrm{G}$ on Mpc scales in the present epoch, when a charged field carrying an elementary charge with mass of order the Hubble scale or smaller exists in the Lagrangian. Similar constraints from the Schwinger effect apply for other magnetogenesis mechanisms.

KEYwords: Cosmology of Theories beyond the SM, Nonperturbative Effects, Electromagnetic Processes and Properties

ARXIV EPRINT: 1408.4141 


\section{Contents}

1 Introduction 1

2 Schwinger effect in de Sitter space 3

2.1 Pair production rate 5

2.2 Induced current and conductivity 8

2.2.1 Strong electric force: $|e E| \gg H^{2} \quad 12$

2.2.2 Weak electric force: $|e E| \ll H^{2} \quad 14$

$\begin{array}{lll}2.2 .3 & \text { Comments on very light or massless scalars } & 15\end{array}$

3 Constraints on inflationary magnetogenesis 16

$\begin{array}{lll}3.1 \text { Model } & 17\end{array}$

$\begin{array}{lll}3.2 & \text { In the absence of charged fields } & 18\end{array}$

$\begin{array}{ll}3.3 & \text { Constraints from Schwinger effect } 20\end{array}$

4 Conclusions $\quad 25$

$\begin{array}{ll}\text { A Some properties of Whittaker functions } & 27\end{array}$

$\begin{array}{ll}\text { B Computation of the current before regularization } & 28\end{array}$

\section{Introduction}

Particle creation by a time dependent background happens in various situations. The wellknown example is the production of charged particles under strong electric fields [1-5], arising from a time dependent vector potential, as studied by Schwinger. Similar phenomena are also seen in curved spacetimes, where time dependent gravitational backgrounds produce particles. Such gravitational effects are particularly important for cosmology, as the large-scale structure in the universe can be seeded by the accelerated expansion during the inflationary epoch (see e.g. [6] for a review). In addition to the cosmic structures, the magnetic fields in our universe may also have a cosmological origin. The possibility of electromagnetic fields existing in the early universe motivates us to look into effects induced by electromagnetic fields in curved spacetimes. Recently, the Schwinger effect in two-dimensional de Sitter (dS) space was studied in [7] (see also [8]). The authors found behaviors quite different from those in flat space; for instance, a large current is induced under weak electric fields, when the mass of the charged particle is much smaller than the Hubble scale, a phenomenon dubbed as "hyperconductivity".

With cosmological applications in mind, in this paper we explore the Schwinger process in four-dimensional dS space. Considering a charged scalar, both the electric and gravitational background fields give rise to the production of the scalar particles. Our strategy 
is to study the expectation value of the induced current, as in [7, 9-14]. This allows us to analyze cases where even the adiabatic vacuum does not exist in the asymptotic future, in other words, regimes where the scalar mass and electric force are much smaller than the Hubble scale and thus the scalar excitations are not well described as particles. Upon computing the expectation value of the current whose formal expression has ultraviolet divergences, we use the method of adiabatic subtraction [15-20] in order to remove the infinities. Under strong electric fields, i.e. $|e E| \gg H^{2}$, the induced current $J$ is obtained as

$$
J \propto \frac{e^{3} E^{2}}{H} e^{-\frac{\pi m^{2}}{|e E|}},
$$

where $H$ is the Hubble rate, $E$ the electric field amplitude, $e$ the scalar charge, and $m$ is the scalar mass. Such a behavior of the current is analogous to that from the Schwinger process in flat space $[13,14]$. On the other hand, with weak electric fields, i.e. $|e E| \ll H^{2}$, we find that the current depends linearly on $E$,

$$
J \propto \frac{e^{2} E H^{3}}{m^{2}} .
$$

Thus we confirm that for small mass, a four-dimensional dS also induces large current from weak electric fields. However, unlike in the two-dimensional case [7] where the current under weak electric fields is exponentially suppressed for massive scalars, in four-dimensions the scaling (1.2) holds for arbitrary masses. Therefore charged massive scalars can also give rise to non-negligible conductivity in a dS universe under weak electric fields.

After analyzing the Schwinger effect in de Sitter space, we move on to apply the results to constrain electromagnetic fields in the early universe. We particularly focus on cosmological scenarios for generating large-scale magnetic fields during the inflationary epoch $[21,22]$. Inflationary magnetogenesis is generically accompanied by the generation of large electric fields as well [23-27], which gives rise to a current via the Schwinger process. When the induced current becomes large, its backreaction to the Maxwell fields becomes non-negligible and can prevent any further generation of the magnetic fields. Such considerations allow us to constrain models of inflationary magnetogenesis from the Schwinger effect. Focusing on models where the electromagnetic fields are generated by a time dependent coupling on the Maxwell kinetic term (of the form $I(t)^{2} F_{\mu \nu} F^{\mu \mu}[22]$ ), we find that the Schwinger effect presents a serious obstacle to generating primordial magnetic fields during inflation. For example, having a field in the action that carries an electric charge of order the elementary charge and mass of order the Hubble scale or smaller, the Schwinger effect prohibits inflationary magnetogenesis from producing magnetic fields larger than $10^{-30} \mathrm{G}$ on Mpc scales in the current universe. The bound depends on the charges and masses of the fields in the action, however the Schwinger effect is shown to pose a major challenge for generating magnetic fields as large as $10^{-15} \mathrm{G}$, which is the lower bound on the extragalactic magnetic fields suggested by the recent gamma ray observations [28-34].

This paper is organized as follows: we investigate the Schwinger effect in a fourdimensional dS space in section 2. After explaining the setup, we carry out the usual 
Bogoliubov calculations in subsection 2.1, limiting ourselves to the regime of $|e E|, m^{2} \gg$ $H^{2}$ so that the adiabatic vacuum exists in the asymptotic future. The reader interested in the induced current/conductivity or constraints on magnetogenesis can skip this subsection, as the results obtained from the Bogoliubov calculation will only be used upon making semiclassical estimates in later discussions. In subsection 2.2, we compute the expectation value of the current, using the method of adiabatic regularization. The behavior of the induced current is studied in various limits, including regimes where the Hubble scale is much larger than the electric force and the scalar mass. We then apply the results to constrain inflationary magnetogenesis in section 3 . This section can also be considered as providing discussions on the issue of backreaction to the background electric field, in the context of magnetogenesis scenarios. Finally, we conclude in section 4 .

Throughout this paper, we take the principal values $-\pi \leq \arg \varpi \leq \pi$ for the phase of complex numbers $\varpi$.

\section{Schwinger effect in de Sitter space}

In order to study the Schwinger process in a four-dimensional dS space, we analyze QED coupled to a charged complex scalar:

$$
S=\int d^{4} x \sqrt{-g}\left\{-g^{\mu \nu}\left(\partial_{\mu}-i e A_{\mu}\right) \varphi^{*}\left(\partial_{\nu}+i e A_{\nu}\right) \varphi-m^{2} \varphi^{*} \varphi-\frac{1}{4} F_{\mu \nu} F^{\mu \nu}\right\},
$$

where $F_{\mu \nu}=\partial_{\mu} A_{\nu}-\partial_{\nu} A_{\mu}$. The background spacetime is fixed to dS,

$$
d s^{2}=a(\tau)^{2}\left(-d \tau^{2}+d x^{2}+d y^{2}+d z^{2}\right),
$$

where the conformal time $\tau$ is expressed in terms of the constant Hubble parameter as

$$
\tau=-\frac{1}{a H}<0, \quad H=\frac{d a}{a^{2} d \tau}=\text { const. }
$$

Here we have taken $\tau \rightarrow 0^{-}$to denote the asymptotic future. We use Greek letters for the spacetime indices $\mu, \nu=\tau, x, y, z$, and Latin letters for spatial indices $i, j=x, y, z$.

In order to describe a constant and uniform electric field, we consider a vector potential of the form

$$
A_{\mu}=\frac{E}{H^{2} \tau} \delta_{\mu}^{z}, \quad E=\text { const. }
$$

Then a comoving observer with 4 -velocity $u^{\mu}\left(u^{i}=0, u_{\mu} u^{\mu}=-1\right)$ measures an electric field along the $z$-direction,

$$
E_{\mu}=u^{\nu} F_{\mu \nu}=a E \delta_{\mu}^{z},
$$

with a constant field strength $E_{\mu} E^{\mu}=E^{2}$.

The equation of motion of $\varphi$ under the time dependent background is

$$
\varphi^{\prime \prime}+2 \frac{a^{\prime}}{a} \varphi^{\prime}-\partial_{i} \partial_{i} \varphi-2 i e A_{z} \partial_{z} \varphi+e^{2} A_{z}^{2} \varphi+a^{2} m^{2} \varphi=0,
$$


where the prime represents a $\tau$-derivative, and the sum over repeated spatial indices is implied irrespective of their positions. Upon quantizing the scalar field $\varphi$ under the time dependent background, let us redefine the field as

$$
q=a \varphi
$$

then the conjugate momenta are obtained from the action $S=\int d^{4} x \mathcal{L}$ in (2.1) as

$$
\Pi=\frac{\partial \mathcal{L}}{\partial q^{\prime}}=q^{*}-\frac{a^{\prime}}{a} q^{*}, \quad \Pi^{*}=\frac{\partial \mathcal{L}}{\partial q^{\prime *}}=q^{\prime}-\frac{a^{\prime}}{a} q .
$$

We promote $q, q^{*}$, and their conjugate momenta into operators,

$$
\begin{aligned}
q(\tau, \boldsymbol{x}) & =\frac{1}{(2 \pi)^{3}} \int d^{3} k\left\{a_{\boldsymbol{k}} q_{\boldsymbol{k}}(\tau) e^{i \boldsymbol{k} \cdot \boldsymbol{x}}+b_{\boldsymbol{k}}^{\dagger} q_{-\boldsymbol{k}}^{*}(\tau) e^{-i \boldsymbol{k} \cdot \boldsymbol{x}}\right\}, \\
q^{\dagger}(\tau, \boldsymbol{x}) & =\frac{1}{(2 \pi)^{3}} \int d^{3} k\left\{a_{\boldsymbol{k}}^{\dagger} q_{\boldsymbol{k}}^{*}(\tau) e^{-i \boldsymbol{k} \cdot \boldsymbol{x}}+b_{\boldsymbol{k}} q_{-\boldsymbol{k}}(\tau) e^{i \boldsymbol{k} \cdot \boldsymbol{x}}\right\},
\end{aligned}
$$

and assign the commutation relations

$$
\begin{aligned}
& {\left[a_{\boldsymbol{k}}, a_{\boldsymbol{p}}^{\dagger}\right]=\left[b_{\boldsymbol{k}}, b_{\boldsymbol{p}}^{\dagger}\right]=(2 \pi)^{3} \delta^{(3)}(\boldsymbol{k}-\boldsymbol{p})} \\
& {\left[a_{\boldsymbol{k}}, a_{\boldsymbol{p}}\right]=\left[b_{\boldsymbol{k}}, b_{\boldsymbol{p}}\right]=\left[a_{\boldsymbol{k}}, b_{\boldsymbol{p}}\right]=\left[a_{\boldsymbol{k}}, b_{\boldsymbol{p}}^{\dagger}\right]=\cdots=0}
\end{aligned}
$$

as well as

$$
\begin{aligned}
{[q(\tau, \boldsymbol{x}), \Pi(\tau, \boldsymbol{y})] } & =\left[q^{\dagger}(\tau, \boldsymbol{x}), \Pi^{\dagger}(\tau, \boldsymbol{y})\right]=i \delta^{(3)}(\boldsymbol{x}-\boldsymbol{y}), \\
{[q(\tau, \boldsymbol{x}), q(\tau, \boldsymbol{y})] } & =[\Pi(\tau, \boldsymbol{x}), \Pi(\tau, \boldsymbol{y})]=\left[q(\tau, \boldsymbol{x}), q^{\dagger}(\tau, \boldsymbol{y})\right]=\left[q(\tau, \boldsymbol{x}), \Pi^{\dagger}(\tau, \boldsymbol{y})\right]=\cdots=0
\end{aligned}
$$

The relations (2.11) follow from (2.10) when the mode function $q_{\boldsymbol{k}}$ satisfies the normalization condition:

$$
q_{\boldsymbol{k}} q_{\boldsymbol{k}}^{*}-q_{\boldsymbol{k}}^{*} q_{\boldsymbol{k}}^{\prime}=i
$$

The mode functions obey the equation of motion (cf. (2.6)) taking the form of

$$
q_{\boldsymbol{k}}^{\prime \prime}+\omega_{\boldsymbol{k}}^{2} q_{\boldsymbol{k}}=0
$$

where the effective frequency squared $\omega_{\boldsymbol{k}}^{2}$ is

$$
\begin{aligned}
\omega_{\boldsymbol{k}}^{2} & =\left(k_{z}+e A_{z}\right)^{2}+k_{x}^{2}+k_{y}^{2}+a^{2} m^{2}-\frac{a^{\prime \prime}}{a} \\
& =\frac{1}{\tau^{2}}\left(\frac{e^{2} E^{2}}{H^{4}}+\frac{m^{2}}{H^{2}}-2\right)+\frac{2}{\tau} \frac{k_{z} e E}{H^{2}}+k^{2} .
\end{aligned}
$$

Here, $k=\left(k_{x}^{2}+k_{y}^{2}+k_{z}^{2}\right)^{1 / 2}$. In the asymptotic past $\tau \rightarrow-\infty$, the frequency is $\omega_{\boldsymbol{k}}^{2} \simeq k^{2}$, and thus $q_{\boldsymbol{k}}$ is a sum of plane waves. On the other hand, in the asymptotic future $\tau \rightarrow 0$, the frequency approaches

$$
\omega_{\boldsymbol{k}}^{2} \simeq \frac{1}{\tau^{2}}\left(\frac{e^{2} E^{2}}{H^{4}}+\frac{m^{2}}{H^{2}}-2\right),
$$


whose rate of change is

$$
\left(\frac{\omega_{\boldsymbol{k}}^{\prime}}{\omega_{\boldsymbol{k}}^{2}}\right)^{2} \simeq\left(\frac{e^{2} E^{2}}{H^{4}}+\frac{m^{2}}{H^{2}}-2\right)^{-1}, \quad \frac{\omega_{\boldsymbol{k}}^{\prime \prime}}{\omega_{\boldsymbol{k}}^{3}} \simeq 2\left(\frac{e^{2} E^{2}}{H^{4}}+\frac{m^{2}}{H^{2}}-2\right)^{-1} .
$$

Thus when $e^{2} E^{2} / H^{4}+m^{2} / H^{2}$ is much larger than unity, then $q_{\boldsymbol{k}}$ in the asymptotic future is well approximated by a WKB solution, in other words, there exists an adiabatic vacuum for $\varphi$.

Let us now introduce the variables

$$
z \equiv 2 k i \tau, \quad \kappa \equiv-i \frac{k_{z}}{k} \frac{e E}{H^{2}}, \quad \mu^{2} \equiv \frac{9}{4}-\frac{e^{2} E^{2}}{H^{4}}-\frac{m^{2}}{H^{2}},
$$

where $z$ and $\kappa$ are purely imaginary, while $\mu$ is either real or purely imaginary. Then the equation of motion (2.13) is rewritten as

$$
\frac{d^{2} q_{k}}{d z^{2}}+\left\{\frac{1}{z^{2}}\left(\frac{1}{4}-\mu^{2}\right)+\frac{\kappa}{z}-\frac{1}{4}\right\} q_{k}=0 .
$$

Solutions of this equation are the Whittaker functions $W_{\kappa, \mu}(z), M_{\kappa, \mu}(z)$, whose basic properties are laid out in appendix A. From the limiting form of $W_{\kappa, \mu}(z)$ as $|z| \rightarrow \infty$ shown in (A.7), we see that the function $W_{\kappa, \mu}(z)$ represents the positive frequency solution in the asymptotic past. Thus we choose the mode function as

$$
q_{k}=\frac{e^{i \kappa \pi / 2}}{\sqrt{2 k}} W_{\kappa, \mu}(z)
$$

where the normalization is set from the condition (2.12), up to an arbitrary phase.

\subsection{Pair production rate}

Let us now evaluate the pair creation rate of the charged scalar particles. In this subsection we limit ourselves to cases where

$$
\frac{e^{2} E^{2}}{H^{4}}+\frac{m^{2}}{H^{2}} \gg 1
$$

so that there exists an adiabatic vacuum for the charged scalar in the asymptotic future. (See discussions around (2.17).) Then the scalar excitations can be interpreted as creation of particles at some intermediate time, and the production rate can be obtained by computing the Bogoliubov coefficients. Under (2.21), $\mu$ is purely imaginary, and we take $\arg \mu=\pi / 2$, i.e.

$$
\mu=i|\mu|
$$

throughout this subsection. In order to study the particle excitations at late times, let us now rewrite the mode function in terms of $M_{\kappa, \mu}(z)$ (see also appendix A, and note especially that $2 \mu$ is not an integer in this subsection),

$$
q_{\boldsymbol{k}}=\frac{e^{-|\mu| \pi / 2}}{2 \sqrt{k|\mu|}}\left\{\alpha_{\boldsymbol{k}} M_{\kappa, \mu}(z)+\beta_{\boldsymbol{k}}\left(M_{\kappa, \mu}(z)\right)^{*}\right\} .
$$


The coefficients $\alpha_{\boldsymbol{k}}$ and $\beta_{\boldsymbol{k}}$ should satisfy

$$
\left|\alpha_{\boldsymbol{k}}\right|^{2}-\left|\beta_{\boldsymbol{k}}\right|^{2}=1
$$

from the normalization condition (2.12). Here we remark that,

$$
\frac{e^{-|\mu| \pi / 2}}{2 \sqrt{k|\mu|}} M_{\kappa, \mu}(z)
$$

represents the positive frequency solution in the asymptotic future. From the limiting form of $M_{\kappa, \mu}(z)$ as $z \rightarrow 0$ shown in (A.8), it can be checked that the solution (2.25) coincides, up to a time independent phase, with the WKB solution in the $\tau \rightarrow 0$ limit:

$$
\frac{1}{\sqrt{2\left|\omega_{\boldsymbol{k}}\right|}} \exp \left\{-i \int^{\tau} d \tau\left|\omega_{\boldsymbol{k}}\right|\right\} \simeq(2|\mu|)^{-1 / 2}(-\tau)^{i|\mu|+1 / 2} e^{i \cdot \text { const. }}
$$

Here, upon obtaining the right hand side, we have used (2.16) and $|\mu|^{2} \gg 1$.

The Bogoliubov coefficients $\alpha_{\boldsymbol{k}}$ and $\beta_{\boldsymbol{k}}$ are obtained from (2.20) and (2.23) by using the formula (A.5) as

$$
\alpha_{\boldsymbol{k}}=(2|\mu|)^{1 / 2} e^{(i \kappa+|\mu|) \pi / 2} \frac{\Gamma(-2 \mu)}{\Gamma\left(\frac{1}{2}-\mu-\kappa\right)}, \quad \beta_{\boldsymbol{k}}=-i(2|\mu|)^{1 / 2} e^{(i \kappa-|\mu|) \pi / 2} \frac{\Gamma(2 \mu)}{\Gamma\left(\frac{1}{2}+\mu-\kappa\right)} .
$$

Choosing the vacuum $|\overline{0}\rangle$ in the asymptotic future by $\bar{a}_{\boldsymbol{k}}|\overline{0}\rangle=\bar{b}_{\boldsymbol{k}}|\overline{0}\rangle=0$ for ${ }^{\forall} \boldsymbol{k}$, where

$$
\bar{a}_{\boldsymbol{k}}=\alpha_{\boldsymbol{k}} a_{\boldsymbol{k}}+\beta_{\boldsymbol{k}}^{*} b_{-\boldsymbol{k}}^{\dagger}, \quad \bar{b}_{\boldsymbol{k}}=\beta_{-\boldsymbol{k}}^{*} a_{-\boldsymbol{k}}^{\dagger}+\alpha_{-\boldsymbol{k}} b_{\boldsymbol{k}},
$$

the number of created particles in the vacuum $|\overline{0}\rangle$ with charge $\mp e$ and comoving wave number $\pm \boldsymbol{k}$ per comoving three-volume is

$$
\frac{\left\langle\overline{0}\left|a_{\boldsymbol{k}}^{\dagger} a_{\boldsymbol{k}}\right| \overline{0}\right\rangle}{(2 \pi)^{3} \int d^{3} x}=\frac{\left\langle\overline{0}\left|b_{-\boldsymbol{k}}^{\dagger} b_{-\boldsymbol{k}}\right| \overline{0}\right\rangle}{(2 \pi)^{3} \int d^{3} x}=\frac{\left|\beta_{\boldsymbol{k}}\right|^{2}}{(2 \pi)^{3}}=\frac{e^{2 i \kappa \pi}+e^{-2|\mu| \pi}}{2(2 \pi)^{3} \sinh (2|\mu| \pi)} .
$$

Integrating this expression over all wave modes gives a divergent result:

$$
\frac{1}{(2 \pi)^{3}} \int d^{3} k\left|\beta_{\boldsymbol{k}}\right|^{2}=\frac{1}{(2 \pi)^{3} \sinh (2|\mu| \pi)}\left\{\frac{H^{2}}{e E} \sinh \left(\frac{2 \pi e E}{H^{2}}\right)+2 \pi e^{-2|\mu| \pi}\right\} \int_{0}^{\infty} d k k^{2},
$$

since it denotes the number of particle pairs produced from the infinite past to the infinite future. Instead of the sum over all times, we are rather interested in the produced number of pairs per unit time.

Under the condition (2.21), the rate of change of the effective frequency $\omega_{\boldsymbol{k}}(2.15)$ is tiny in both the asymptotic past and future, and thus there exist adiabatic vacua for the charged scalar. Here, let us estimate the time of particle creation by analyzing when the adiabaticity is violated, i.e., when $\omega_{\boldsymbol{k}}$ changes quickly, by studying how $\left|\omega_{\boldsymbol{k}}^{\prime} / \omega_{\boldsymbol{k}}^{2}\right|$ grows in time. The quantity $\left|\omega_{\boldsymbol{k}}^{\prime} / \omega_{\boldsymbol{k}}^{2}\right|$ vanishes in the asymptotic past, and it approaches the value (2.17) in the asymptotic future. Depending on the parameter values, the time evolution of $\left|\omega_{\boldsymbol{k}}^{\prime} / \omega_{\boldsymbol{k}}^{2}\right|$ may or may not exhibit peaks in the intermediate times; e.g. for $k_{z} e E<0$, then $\left|\omega_{\boldsymbol{k}}^{\prime} / \omega_{\boldsymbol{k}}^{2}\right|$ can 
just monotonically grow in time. In such cases where $\left|\omega_{\boldsymbol{k}}^{\prime} / \omega_{\boldsymbol{k}}^{2}\right|$ does not exhibit peaks, we can instead focus on when $\left|\omega_{\boldsymbol{k}}^{\prime} / \omega_{\boldsymbol{k}}^{2}\right|$ comes close to taking the asymptotic value (2.17). It can be checked that, for parameter sets that satisfy $-\mu^{2} \gg 1$, it is around the time

$$
\tau \sim-\frac{1}{k}\left(|\mu|^{2}+\frac{1}{4}\right)^{1 / 2}
$$

when the quantity $\left|\omega_{\boldsymbol{k}}^{\prime} / \omega_{\boldsymbol{k}}^{2}\right|$ exhibits peaks, or approaches closely to its maximum value. ${ }^{1}$

Thus we make use of the rough estimate (2.31) and translate the $k$-integral in (2.30) into a time integral,

$$
\frac{1}{(2 \pi)^{3}} \int d^{3} k\left|\beta_{\boldsymbol{k}}\right|^{2}=\frac{\left(|\mu|^{2}+\frac{1}{4}\right)^{3 / 2}}{(2 \pi)^{3} \sinh (2|\mu| \pi)}\left\{\frac{H^{2}}{e E} \sinh \left(\frac{2 \pi e E}{H^{2}}\right)+2 \pi e^{-2|\mu| \pi}\right\} \int_{-\infty}^{0} d \tau(a H)^{4} .
$$

By looking at the produced number of pairs within $d \tau$, and dividing by $a^{4}$, we arrive at the pair production rate, i.e., the number of pairs produced per unit physical four-volume,

$$
\Gamma=\frac{H^{4}}{(2 \pi)^{3}} \frac{\left(|\mu|^{2}+\frac{1}{4}\right)^{3 / 2}}{\sinh (2|\mu| \pi)}\left\{\frac{H^{2}}{e E} \sinh \left(\frac{2 \pi e E}{H^{2}}\right)+2 \pi e^{-2|\mu| \pi}\right\} .
$$

Since the rate $\Gamma$ is independent of time, the physical number density $n$ of pairs at time $\tau$ is easily computed as

$$
n=\frac{1}{a(\tau)^{3}} \int_{-\infty}^{\tau} d \tilde{\tau} a(\tilde{\tau})^{4} \Gamma=\frac{\Gamma}{3 H} .
$$

The fact that $n$ is a constant indicates that the Schwinger and gravitational particle creation balances against the dilution of the number density due to the expansion of the universe. One sees that, when the mass and/or the electric field are large enough to satisfy the condition (2.21), the $\varphi$ population is always dominated by the particles created within a Hubble time.

The vacuum persistence probability can also be computed in a similar fashion from

$$
|\langle\overline{0} \mid 0\rangle|^{2}=\exp \left\{-\frac{\int d^{3} x}{(2 \pi)^{3}} \int d^{3} k \ln \left(1+\left|\beta_{\boldsymbol{k}}\right|^{2}\right)\right\} .
$$

Here we further assume $\frac{m^{2}}{H^{2}} \geq \frac{9}{4}$ in addition to (2.21), and use the formula for the dilogarithm,

$$
-\int_{0}^{z} d s \frac{\ln (1-s)}{s}=\sum_{j=1}^{\infty} \frac{z^{j}}{j^{2}}, \quad \text { for }|z| \leq 1,
$$

for integrating over the angular direction $k_{z} / k$. Then, converting the $k$-integral into the time integral using (2.31), one can obtain the vacuum decay rate $\Upsilon_{\text {vac }}$ :

$$
|\langle\overline{0} \mid 0\rangle|^{2}=\exp \left\{-\int d^{3} x d \tau a^{4} \Upsilon_{\mathrm{vac}}\right\}
$$

\footnotetext{
${ }^{1}$ The violation of the adiabaticity can also be studied in a different frame; by redefining the field and time as $q_{\boldsymbol{k}}=a^{m} \chi_{\boldsymbol{k}}, d \tau=a^{2 m} d s$, such that the form of the equation of motion (2.13) is preserved. The detailed behavior of $\left|\omega_{\boldsymbol{k}}^{\prime} / \omega_{\boldsymbol{k}}^{2}\right|$ (e.g., whether it peaks at a certain time, or monotonically grows) depends on the frame, however we remark that the order-of-magnitude estimate (2.31) of when $\left|\omega_{\boldsymbol{k}}^{\prime} / \omega_{\boldsymbol{k}}^{2}\right|$ approaches its maximum value is independent of the choice of the frame.
} 
as a series of the form,

$$
\Upsilon_{\mathrm{vac}}=\frac{H^{4}}{(2 \pi)^{3}}\left(|\mu|^{2}+\frac{1}{4}\right)^{3 / 2} \sum_{j=1}^{\infty}\left\{\frac{(-1)^{j+1}}{j^{2}} \frac{2 H^{2}}{e E} e^{-2 j|\mu| \pi} \sinh \left(\frac{2 j \pi e E}{H^{2}}\right)+\frac{2 \pi}{j} e^{-4 j|\mu| \pi}\right\} .
$$

Let us close this section by studying the limit where the Hubble parameter is much smaller than the mass and electric field strength. Taking $H \rightarrow 0$ in the above expressions gives

$$
\begin{aligned}
\lim _{H \rightarrow 0} \Gamma & =\frac{(e E)^{2}}{(2 \pi)^{3}} \exp \left(-\frac{\pi m^{2}}{|e E|}\right), \\
\lim _{H \rightarrow 0} \Upsilon_{\mathrm{vac}} & =\sum_{j=1}^{\infty} \frac{(-1)^{j+1}}{j^{2}} \frac{(e E)^{2}}{(2 \pi)^{3}} \exp \left(-\frac{j \pi m^{2}}{|e E|}\right),
\end{aligned}
$$

reproducing the familiar results for Schwinger pair production in Minkowski space [3-5] (see also [13] for a recent analysis).

\subsection{Induced current and conductivity}

Once produced, the charged scalar particles move under the electric field and thus give rise to a current as well as a conductivity. The results obtained in subsection 2.1 can be used to estimate the induced current via $|J| \sim|2 e n v|$, where $v$ is the velocity of the particles. Such a semiclassical approach provides good approximations in some parameter regions (as we will see later), but not in general. In particular, computing the contribution only from the created particles is not enough, as such a naive picture violates local charge conservation [7]. Moreover, the analyses in the previous subsection were limited to cases where the mass and/or the electric force are sufficiently larger than the Hubble scale, cf. (2.21).

In this subsection we do not impose the condition (2.21), and directly compute the expectation value of the conserved current, ${ }^{2}$

$$
J_{\mu}=\frac{i e}{2}\left\{\varphi^{\dagger}\left(\partial_{\mu}+i e A_{\mu}\right) \varphi-\varphi\left(\partial_{\mu}-i e A_{\mu}\right) \varphi^{\dagger}\right\}+\text { h.c. }
$$

in the vacuum state $|0\rangle$ defined by $a_{\boldsymbol{k}}|0\rangle=b_{\boldsymbol{k}}|0\rangle=0$ for ${ }^{\forall} \boldsymbol{k}$, cf. (2.9). Under the electric field along the $z$-direction, the expectation value vanishes except for its $z$-component,

$$
\left\langle J_{z}\right\rangle=-\frac{2 e}{(2 \pi)^{3} a^{2}} \int d^{3} k\left(k_{z}+e A_{z}\right)\left|q_{\boldsymbol{k}}\right|^{2},
$$

where the mode function is given in (2.20). However this expectation value diverges, as can be seen from the limiting form of $q_{\boldsymbol{k}}$ as $k \rightarrow \infty$ shown in (A.7). In order to explicitly see the divergence, let us first compute the integral by imposing a cutoff $\zeta$ on $k$,

$$
\left\langle J_{z}\right\rangle=-\lim _{\zeta \rightarrow \infty} \frac{2 e}{(2 \pi)^{2} a^{2}} \int_{0}^{\zeta} d k k^{2} \int_{-1}^{1} d r\left(k r+e A_{z}\right) \frac{e^{i \kappa \pi}}{2 k}\left|W_{\kappa, \mu}(z)\right|^{2},
$$

\footnotetext{
${ }^{2}$ In terms of the current $J_{\mu}(2.42)$, the Maxwell equation is written as
}

$$
\nabla^{\nu} F_{\mu \nu}=J_{\mu} .
$$


where we have introduced

$$
r=\frac{k_{z}}{k} \text {. }
$$

The integral is carried out in appendix B, yielding

$$
\begin{aligned}
\left\langle J_{z}\right\rangle= & \frac{e a H^{3}}{(2 \pi)^{2}} \lim _{\zeta \rightarrow \infty}\left[\frac{2 \lambda}{3}\left(\frac{\zeta}{a H}\right)^{2}+\frac{\lambda}{3} \ln \left(\frac{2 \zeta}{a H}\right)-\frac{25 \lambda}{36}+\frac{\mu^{2} \lambda}{3}+\frac{\lambda^{3}}{15}\right. \\
& +\frac{45+4 \pi^{2}\left(-2+3 \lambda^{2}+2 \mu^{2}\right)}{12 \pi^{3}} \frac{\mu \cosh (2 \pi \lambda)}{\lambda \sin (2 \pi \mu)}-\frac{45+8 \pi^{2}\left(-1+9 \lambda^{2}+\mu^{2}\right)}{24 \pi^{4}} \frac{\mu \sinh (2 \pi \lambda)}{\lambda^{2} \sin (2 \pi \mu)} \\
& +\operatorname{Re}\left\{\int_{-1}^{1} d r \frac{i \lambda}{16 \sin (2 \pi \mu)}\left(-1+4 \mu^{2}+\left(7+12 \lambda^{2}-12 \mu^{2}\right) r^{2}-20 \lambda^{2} r^{4}\right)\right. \\
& \left.\left.\times\left(\left(e^{2 \pi r \lambda}+e^{2 \pi i \mu}\right) \psi\left(\frac{1}{2}+\mu+i r \lambda\right)-\left(e^{2 \pi r \lambda}+e^{-2 \pi i \mu}\right) \psi\left(\frac{1}{2}-\mu+i r \lambda\right)\right)\right\}\right],
\end{aligned}
$$

where $\psi(z)=\Gamma^{\prime}(z) / \Gamma(z)$ is the digamma function, and $\lambda$ is defined as

$$
\lambda=\frac{e E}{H^{2}}
$$

We thus see that the expectation value of the current has quadratic and logarithmic divergences. Let us also remark that some of the terms in (2.46) blow up when $\mu=0,1 / 2, \ldots$. However their sum does not necessarily diverge as $\mu$ approaches such values, and thus the finite part of (2.46) (i.e. terms without $\zeta$ ) is well-behaved.

In order to regularize the divergences, we use the method of adiabatic subtraction [1520]. The idea here is to compute quantities in the limit of slow variation of the background, then subtract their contributions from the formal expressions to obtain a finite result. (See also works [9-12] which applied adiabatic regularization to the analysis of Schwinger effect in flat space.) Let us start by considering a mode function with a WKB form,

$$
q_{\boldsymbol{k}}(\tau)=\frac{1}{\sqrt{2 W_{\boldsymbol{k}}(\tau)}} \exp \left\{-i \int^{\tau} d \tilde{\tau} W_{\boldsymbol{k}}(\tilde{\tau})\right\}
$$

which is an exact solution of the equation of motion (2.13) if the function $W_{\boldsymbol{k}}$ satisfies

$$
W_{k}^{2}=\omega_{k}^{2}+\frac{3}{4}\left(\frac{W_{k}^{\prime}}{W_{k}}\right)^{2}-\frac{1}{2} \frac{W_{k}^{\prime \prime}}{W_{k}}
$$

Furthermore, when $W_{\boldsymbol{k}}$ is real and positive, the normalization condition (2.12) is also satisfied. Here, recall from (2.14) that $\omega_{\boldsymbol{k}}^{2}$ takes the form of

$$
\omega_{k}^{2}=\Omega_{k}^{2}-\frac{a^{\prime \prime}}{a}
$$

with

$$
\Omega_{\boldsymbol{k}}=\left\{\left(k_{z}+e A_{z}\right)^{2}+k_{x}^{2}+k_{y}^{2}+a^{2} m^{2}\right\}^{1 / 2} .
$$

Hereafter, let us assume the mass to be nonzero, i.e. $m \neq 0$, so that $\Omega_{\boldsymbol{k}}^{2}$ is positive definite. In order to parameterize the slowness of the evolution of the time dependent background, we 
assign an adiabatic parameter $T^{-1}$ to each time derivative in (2.49) and (2.50); taking $T \rightarrow$ $\infty$ denotes the limit of infinitely slow variation of the background. Then the function $W_{\boldsymbol{k}}^{2}$ can be computed at each order in $T^{-1}$. The solution at leading order is simply

$$
W_{\boldsymbol{k}}^{2}=\Omega_{\boldsymbol{k}}^{2}+\mathcal{O}\left(T^{-2}\right)
$$

Higher order solutions can be obtained by recursively substituting the results into the right hand side of (2.49); up to adiabatic order $T^{-2}$ we obtain

$$
W_{k}^{2}=\omega_{k}^{2}+\frac{3}{4}\left(\frac{\Omega_{k}^{\prime}}{\Omega_{k}}\right)^{2}-\frac{1}{2} \frac{\Omega_{\boldsymbol{k}}^{\prime \prime}}{\Omega_{\boldsymbol{k}}}+\mathcal{O}\left(T^{-4}\right),
$$

and so on. We note that our results are not altered by computing the adiabatic subtraction terms in a different frame, where $q_{\boldsymbol{k}}$ and $\tau$ are redefined such that the equation of motion preserves the form of (2.13) (cf. Footnote 1).

In the following we expand the current in terms of $T^{-1}$, then the lower order results will be subtracted from the formal expression (2.44). We will see that the adiabatic subtraction up to quadratic order is just enough to remove the divergences, as well as gives results that have the correct behavior in the Minkowski limit. We also remark that, since the formal expression of $\left\langle J_{0}\right\rangle$ vanishes, and also $\left\langle J_{i}\right\rangle$ is homogeneous, it is clear that the adiabatic subtraction does not spoil the current conservation. Detailed discussions on the method of adiabatic subtraction can be found in, e.g., [15-20].

For a real and positive $W_{\boldsymbol{k}}$ (recall from (2.52) that we can have $W_{\boldsymbol{k}} \simeq \Omega_{\boldsymbol{k}}>0$ at the leading order), the current (2.43) is written in terms of the WKB solution (2.48) as

$$
-\frac{2 e}{(2 \pi)^{3} a^{2}} \int d^{3} k\left(k_{z}+e A_{z}\right) \frac{1}{2 W_{\boldsymbol{k}}} .
$$

Expanding this expression up to adiabatic order $T^{-2}$ using (2.53) yields

$$
\begin{aligned}
-\frac{2 e}{(2 \pi)^{3} a^{2}} \int d^{3} k\left(k_{z}+e A_{z}\right) \frac{1}{2 \Omega_{\boldsymbol{k}}}[ & +\frac{1}{2 \Omega_{\boldsymbol{k}}^{2}} \frac{a^{\prime \prime}}{a} \\
& +\frac{1}{4 \Omega_{k}^{4}}\left\{\left(e A_{z}^{\prime}\right)^{2}+\left(k_{z}+e A_{z}\right) e A_{z}^{\prime \prime}+\left(a^{\prime 2}+a a^{\prime \prime}\right) m^{2}\right\} \\
& \left.-\frac{5}{8 \Omega_{k}^{6}}\left\{\left(k_{z}+e A_{z}\right) e A_{z}^{\prime}+a a^{\prime} m^{2}\right\}^{2}+\mathcal{O}\left(T^{-4}\right)\right] .
\end{aligned}
$$

We carry out the integration by imposing a cutoff $\zeta$ on $k$ as in (2.44). After some algebra we obtain

$$
\begin{aligned}
\lim _{\zeta \rightarrow \infty} \frac{e}{(2 \pi)^{2} a^{2}}[ & -\frac{2}{3} e A_{z} \zeta^{2}+\frac{2}{15}\left(e A_{z}\right)^{3}+\frac{1}{3} e A_{z} a^{2} m^{2} \\
& \left.-\frac{1}{6} e A_{z}^{\prime \prime} \ln \left(\frac{2 \zeta}{a m}\right)+\frac{1}{6} \frac{a^{\prime}}{a} e A_{z}^{\prime}-\frac{1}{3} \frac{a^{\prime \prime}}{a} e A_{z}+\frac{2}{9} e A_{z}^{\prime \prime}+\mathcal{O}\left(T^{-4}\right)\right] .
\end{aligned}
$$


Terms of adiabatic order $T^{0}$ shown in the first line contains a quadratic divergence, while the terms of $T^{-2}$ in the second line has a logarithmic divergence. Substituting the expressions for $a(2.3)$ and $A_{z}(2.4)$, we find the adiabatic subtraction terms to be

$$
\lim _{\zeta \rightarrow \infty} \frac{e a H^{3}}{(2 \pi)^{2}}\left[\frac{2 \lambda}{3}\left(\frac{\zeta}{a H}\right)^{2}-\frac{2 \lambda^{3}}{15}-\frac{\lambda}{3} \frac{m^{2}}{H^{2}}+\frac{\lambda}{3} \ln \left(\frac{2 \zeta}{a m}\right)+\frac{\lambda}{18}+\mathcal{O}\left(T^{-4}\right)\right],
$$

where the first three terms arise from the order $T^{0}$ expansion, and the other two terms from order $T^{-2}$. Comparing with the formal expression (2.46), the divergences of the expectation value are seen to be removed by the adiabatic subtraction up to order $T^{-2}$. One can further expand up to order $T^{-4}$, which only gives finite terms. However, as we will see later, subtracting terms of order $T^{-4}$ spoils the behavior of the current in the flat space limit. Therefore, we subtract off terms up to adiabatic order $T^{-2}$ from (2.46) in order to obtain the regularized current, arriving at

$$
\begin{aligned}
\left\langle J_{z}\right\rangle_{\text {reg }}= & \frac{e a H^{3}}{(2 \pi)^{2}}\left[-\frac{2 \lambda^{3}}{15}+\frac{\lambda}{3} \ln \left(\frac{m}{H}\right)\right. \\
& +\frac{45+4 \pi^{2}\left(-2+3 \lambda^{2}+2 \mu^{2}\right)}{12 \pi^{3}} \frac{\mu \cosh (2 \pi \lambda)}{\lambda \sin (2 \pi \mu)}-\frac{45+8 \pi^{2}\left(-1+9 \lambda^{2}+\mu^{2}\right)}{24 \pi^{4}} \frac{\mu \sinh (2 \pi \lambda)}{\lambda^{2} \sin (2 \pi \mu)} \\
& +\operatorname{Re}\left\{\int_{-1}^{1} d r \frac{i \lambda}{16 \sin (2 \pi \mu)}\left(-1+4 \mu^{2}+\left(7+12 \lambda^{2}-12 \mu^{2}\right) r^{2}-20 \lambda^{2} r^{4}\right)\right. \\
& \left.\left.\times\left(\left(e^{2 \pi r \lambda}+e^{2 \pi i \mu}\right) \psi\left(\frac{1}{2}+\mu+i r \lambda\right)-\left(e^{2 \pi r \lambda}+e^{-2 \pi i \mu}\right) \psi\left(\frac{1}{2}-\mu+i r \lambda\right)\right)\right\}\right] .
\end{aligned}
$$

Comparing with the formal expression (2.46), the procedure of adiabatic subtraction has modified the terms in the first line inside the parentheses.

Here it is important to note that the hard cutoff $\zeta$ was introduced in the derivation only for calculational convenience, so that the integrations of the formal expression (2.44) and the adiabatic expansion (2.55) can be performed separately. Instead of using $\zeta$, we could have subtracted the integrand of (2.55) from (2.44) before carrying out the integral, then we would not see any infinities in the calculations. Such a procedure would be preferable for numerical studies.

Before discussing the behavior of (2.58), let us parameterize the amplitude of the current as

$$
\left\langle J_{z}\right\rangle_{\mathrm{reg}}=a J,
$$

where $J$ has mass dimension three. We also define the conductivity $\sigma$ by

$$
\sigma=\frac{J}{E} .
$$

Then one sees from (2.58) that normalized quantities such as

$$
\frac{J}{e H^{3}}, \quad \frac{\sigma}{e^{2} H}=\frac{J}{e H^{3}} \frac{1}{\lambda}
$$


are uniquely fixed by the two parameters $m / H$ and $e E / H^{2}$ (or, equivalently, $\mu$ and $\lambda$ ), representing the mass and electric force relative to the Hubble scale. In particular, $\mathrm{J} / \mathrm{eH}$ and $\sigma / e^{2} H$ are independent of time. Note also that $J / e H^{3}$ and $\sigma / e^{2} H$ are, respectively, odd and even under $\lambda \rightarrow-\lambda$.

In figure 1 we plot $J / e H^{3}$ and $\sigma / e^{2} H$ as functions of $\lambda$, where each curve corresponds to a different choice of mass $m / H$. The solid lines are obtained from the regularized result (2.58). We also show dashed lines denoting semiclassical estimates of the current based on the computations in subsection 2.1, which will be explained later.

The plots show that when $|e E| \gg H^{2}$, the conductivity monotonically grows with increasing $|E|$, and becomes independent of the mass for a sufficiently large $|e E| / H^{2}$. On the other hand, the conductivity is independent of $E$ under weak electric force $|e E| \ll H^{2}$. In particular, for small mass $m \ll H$, the conductivity is strongly enhanced in the weak electric field regime. Let us now study the behavior of $J$ and $\sigma$ in the limiting regimes of $|e E| \gg H^{2}$ and $|e E| \ll H^{2}$, respectively.

\subsubsection{Strong electric force: $|e E| \gg H^{2}$}

In the limit of $|\lambda| \rightarrow \infty$, for a fixed $m / H$, the third and fourth lines of (2.58) approach

$$
\operatorname{Re}\left\{\int d r \cdots\right\} \simeq \frac{2 \lambda^{3}}{15}
$$

and thus largely cancels with the first term in (2.58). Consequently, the cosh term proportional to $\lambda^{2}$ (note that $\mu^{2} \simeq-\lambda^{2}$ ) dominates the current and yields

$$
\begin{gathered}
\frac{J}{H^{3}} \simeq \operatorname{sgn}(\lambda) \frac{e \lambda^{2}}{12 \pi^{3}}=\operatorname{sgn}(E) \frac{1}{12 \pi^{3}} \frac{|e|^{3} E^{2}}{H^{4}}, \\
\frac{\sigma}{H} \simeq \frac{e^{2}|\lambda|}{12 \pi^{3}}=\frac{1}{12 \pi^{3}} \frac{\left|e^{3} E\right|}{H^{2}} .
\end{gathered}
$$

Thus one sees that when $|e E|$ is sufficiently large relative to the Hubble rate $H^{2}$, the current $J$ is quadratic in $E$, and thus $\sigma$ is linear in $E$. These features are also seen in the plots, where all curves converge as $|\lambda| \rightarrow \infty$ and the behavior becomes independent of the mass.

Since the condition (2.21) is satisfied in the regime of $|\lambda| \gg 1$, an adiabatic vacuum exists in the asymptotic future for the $\varphi$ fields. Hence let us try to estimate the current in this regime based on the semiclassical picture of the created particles carrying the charge. Supposing the particles to travel with velocity $v \simeq 1$, then we can estimate the arising current by

$$
J \simeq \operatorname{sgn}(E) 2|e| n=\operatorname{sgn}(E) \frac{2|e| \Gamma}{3 H} .
$$

Here $n$ is the number density of the produced pairs, and we have used (2.34) upon moving to the right hand side. The pair production rate $\Gamma$ was computed in (2.33). Taking the limit of $H \rightarrow 0$ as shown in (2.39), and further taking $m^{2} / e E \rightarrow 0$, one exactly reproduces the result (2.63). 


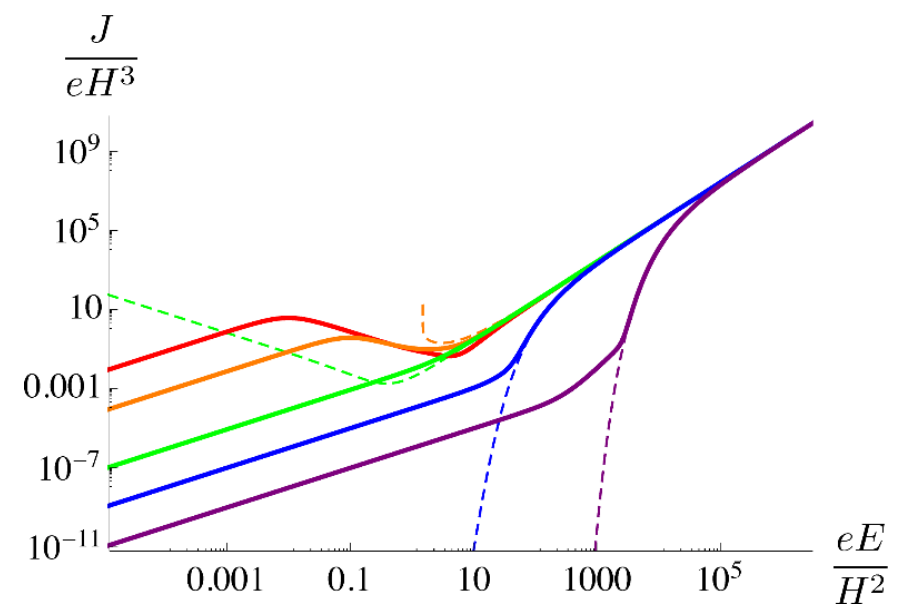

(a) current

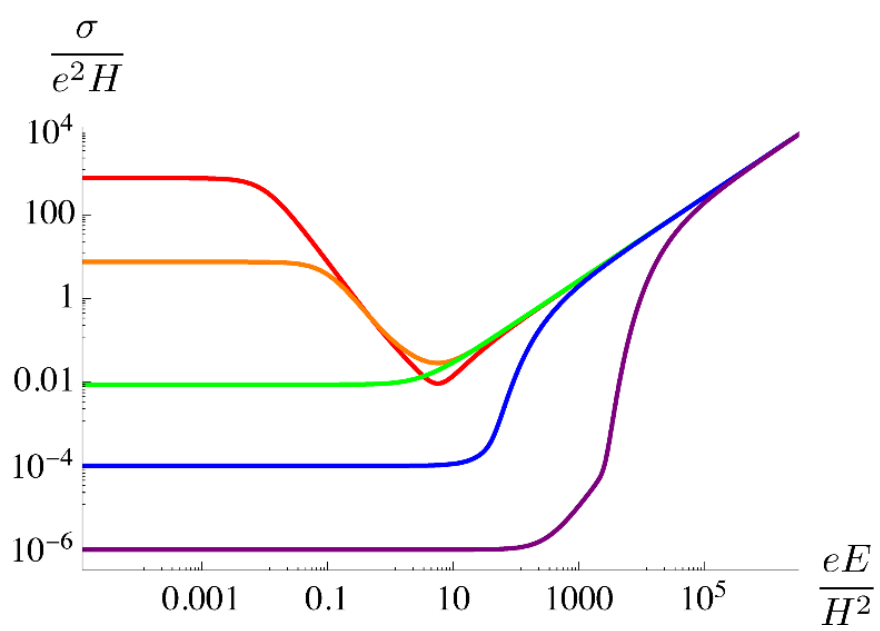

(b) conductivity

Figure 1. Induced current $J$ and conductivity $\sigma$ as a function of the electric field $E$. The displayed quantities are normalized by the Hubble parameter $H$ and charge $e$. Each line is for a different choice of mass, $m / H=0.01$ (red), 0.1(orange), 1.5(green), 10(blue), 100(purple). The semiclassical estimate of the current using $|J| \sim 2|e| n$ is shown as dashed lines, with colors representing the choice of mass.

In figure 1(a) the dashed lines show the semiclassical estimate (2.65) using the production rate $\Gamma$ (2.33). In the regime $|\lambda| \gg 1$, the estimates agree well with the results

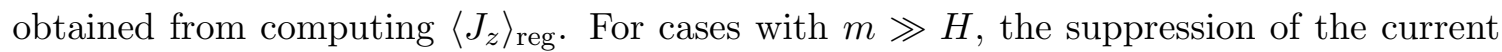
at $|e E| \sim m^{2}$ corresponds to the mass suppression of $\Gamma$ shown in (2.39). Thus we obtain an improved approximation for the current (2.58) at $|\lambda| \gg 1$ as

$$
J \simeq \operatorname{sgn}(E) \frac{1}{12 \pi^{3}} \frac{|e|^{3} E^{2}}{H} e^{-\frac{\pi m^{2}}{|e E|}} .
$$

On the other hand, when $|\lambda| \ll 1$, the semiclassical estimate is seen to break down. Particularly, for light masses $m / H<3 / 2$, the condition (2.21) does not hold and thus the expression (2.33) itself cannot be extended to the $|\lambda| \ll 1$ regime. (We note that the 
semiclassical estimate for $m / H=0.01$ (red dashed line) overlaps with that of $m / H=0.1$ (orange dashed), and thus not seen in the plot.)

The behavior of the current (2.66) in the regime $|\lambda| \gg 1$ corresponds to that induced by the Schwinger effect in a Minkowski space $[13,14]$,

$$
J \sim \operatorname{sgn}(E)|e|^{3} E^{2}\left(t-t_{0}\right) e^{-\frac{\pi m^{2}}{|e E|}}
$$

where $t_{0}$ is the initial time when the electric field is switched on. (The situation here is slightly different from that in (2.66) where the electric field always exist. A finite $t_{0}$ is introduced because, due to the absence of the Hubble dilution, $J$ blows up in a Minkowski space if the electric field existed from the infinite past $t_{0}=-\infty$. This is why the expression (2.66) diverges as $H \rightarrow 0$.) Our results in $\mathrm{dS}$ were obtained by an adiabatic expansion up to quadratic order, however we remark that the adiabatic subtraction at order $T^{-4}$ produces a term that scales as $\lambda^{3}$, which gives a scaling behavior of $J \propto E^{3}$ at $|\lambda| \gg 1$, contrary to (2.67). Therefore we see that the adiabatic subtraction up to order $T^{-2}$ not just removes the divergences, but also produces results with the correct behavior in the Minkowski limit.

Let us also remark that the scaling of $J$ at $|\lambda| \gg 1$ depends on the spacetime dimension. In a two-dimensional dS space, the current induced by strong electric fields scales as $J \propto E$, and thus the conductivity approaches a constant at large $|E|[7]$. As we will see in section 3, $\sigma$ being an increasing function of $E$ in four-dimensions gives rise to stringent constraints on electromagnetic fields in the early universe.

2.2.2 Weak electric force: $|e E| \ll H^{2}$

Taking $\lambda \rightarrow 0$ for a fixed $m / H$, one can check that the current (2.58) becomes dominated by terms linear in $\lambda$, and approximated as

$$
\frac{J}{H^{3}} \simeq \frac{e^{2} E}{24 \pi^{2} H^{2}}\left\{\ln \left(\frac{m^{2}}{H^{2}}\right)+\frac{16 \pi}{3} \frac{\mu_{0}\left(-1+\mu_{0}^{2}\right)}{\sin \left(2 \pi \mu_{0}\right)}-\psi\left(\frac{1}{2}+\mu_{0}\right)-\psi\left(\frac{1}{2}-\mu_{0}\right)\right\},
$$

where

$$
\mu_{0}^{2}=\frac{9}{4}-\frac{m^{2}}{H^{2}}
$$

The expression gets further simplified when $m \gg H$ as

$$
\frac{J}{H^{3}} \simeq \frac{7}{72 \pi^{2}} \frac{e^{2} E}{m^{2}} .
$$

Here the main contributions to the current are given by the terms $\frac{\lambda}{3} \ln \left(\frac{m}{H}\right)$ and $\operatorname{Re}\left\{\int d r \cdots\right\}$ in (2.58), which originate from the adiabatic subtraction (2.57) and formal expression (2.46), respectively. On the other hand, for $m \ll H$, the current is approximated by

$$
\frac{J}{H^{3}} \simeq \frac{3}{4 \pi^{2}} \frac{e^{2} E}{m^{2}} .
$$

The current in this case mainly arises from the terms in (2.58) that involve cosh, sinh, and $\operatorname{Re}\left\{\int d r \cdots\right\}$, which are all contributions originating from the formal expression (2.46). 
Therefore for arbitrary mass, the current and conductivity in the regime $|\lambda| \ll 1$ can be roughly approximated by

$$
\frac{J}{H^{3}} \sim 10^{-2} \times \frac{e^{2} E}{m^{2}}, \quad \frac{\sigma}{H} \sim 10^{-2} \times \frac{e^{2} H^{2}}{m^{2}} .
$$

Here we see that the conductivity is independent of $E$, and takes larger values for a smaller mass ratio $m / H$.

In particular for light mass $m \ll H$, the plot shows that as one decreases $\lambda$, the conductivity grows until it approaches the constant value (2.72). The strong enhancement of the conductivity for small mass scalars under weak electric fields are supported by the infrared contributions to the current: this can be seen from the limiting behavior of the Whittaker function as $z \rightarrow 0$,

$$
W_{\kappa, \mu}(z) \propto z^{\frac{1}{2}-|\operatorname{Re}(\mu)|} .
$$

(Here we note that this expression (2.73) is not valid for an arbitrary set of $\kappa$ and $\mu$, however we use it for the rough estimation in the following.) Hence one sees that the integrand of (2.44) for $\left\langle J_{z}\right\rangle$, in the limit $k \rightarrow 0$, scales as

$$
\propto k^{2(1-|\operatorname{Re}(\mu)|)} .
$$

The simple power counting estimate indicates that the current spectrum diverges in the infrared limit when $|\operatorname{Re}(\mu)|>1$, i.e.,

$$
\frac{e^{2} E^{2}}{H^{4}}+\frac{m^{2}}{H^{2}}<\frac{5}{4}
$$

However, it should also be noted that a nonzero $e E$ or $m$ give $|\operatorname{Re}(\mu)|<\frac{3}{2}$, and thus integrating the spectrum (2.74) down to $k=0$ yields a finite total current. We also remark that the spectrum of the adiabatic subtraction terms in (2.55) are finite in the infrared limit, since $\Omega_{k} \geq a m>0$ for a nonzero mass. Thus we find that the enhanced conductivity at $|\lambda| \ll 1$ for small masses arise from the infrared behavior of the mode function $q_{\boldsymbol{k}}$. The nature of the infrared modes being important should be contrasted to cases $-\mu^{2} \gg 1$ discussed below (2.34), where the newly created particles with $k \sim a H|\mu|$ always dominate the $\varphi$ population.

The constancy of $\sigma$ under weak electric fields, and its strong enhancement for small mass are also seen in a two-dimensional dS space [7]. However there is an important difference worth noting: while in four dimensions the conductivity under weak fields scales as $\sigma \propto m^{-2}$ for all masses, in a two-dimensional dS the scaling $\sigma \propto m^{-2}$ is only for light masses, and the conductivity is exponentially suppressed for $m \gtrsim H$.

\subsubsection{Comments on very light or massless scalars}

In figure 1 we have plotted curves for scalar masses as low as $m / H=0.01$. Cases for even smaller masses have similar behaviors under strong/weak electric fields; the conductivity takes the mass independent value $(2.64)$ at $|\lambda| \gg 1$, while at $|\lambda| \ll 1$ the conductivity grows with decreasing $\lambda$ at a more or less similar rate, until it approaches the constant 
value (2.72) set by the mass. However, we remark that the valley of $\sigma$ in the intermediate regime of $\lambda$ becomes deeper for smaller $m$, and the conductivity can even take negative values at $|\lambda| \sim 1$ for extremely light masses.

For the exactly massless case, i.e $m=0$, one sees that the regularized current (2.58) diverges due to the $\ln (m / H)$ term, which was introduced through the procedure of adiabatic subtraction at the order $T^{-2}$. This divergence originates from $\Omega_{\boldsymbol{k}}$ (2.51) vanishing for $m=0$ at

$$
\left(k_{z}+e A_{z}\right)^{2}+k_{x}^{2}+k_{y}^{2}=0,
$$

and thus blowing up the adiabatic subtraction terms. Note that (2.76) corresponds to the vanishing of the physical momentum

$$
\left(p_{x}, p_{y}, p_{z}\right)=\left(\frac{k_{x}}{a}, \frac{k_{y}}{a}, \frac{k_{z}+e A_{z}}{a}\right) .
$$

These observations suggest that the adiabatic expansion taking $T \rightarrow \infty$ is invalid for zero modes of massless fields, and thus the method of adiabatic regularization may not be applicable for the massless case. (See also discussions in $[15,16]$.) This issue may be resolved by imposing an infrared cutoff on the momentum integral, by considering that in the finite past the dS expansion started, or the electric field was switched on.

We should also mention that, even if a scalar has a tiny bare mass, a Hubble-induced mass can be generated $[35,36]$. See also discussions on effective mass in dS space in, e.g., [37-41]. The charged scalars need to be protected from mass corrections in order for a dS universe to actually possess large conductivity under weak electric fields.

\section{Constraints on inflationary magnetogenesis}

In the previous section we studied the production of charged scalars in a fixed background of a constant electric field and dS expansion. However the backreaction from the produced scalars may become relevant, especially when the induced conductivity is huge. Such considerations are important upon discussing the aftermath of the Schwinger process, but can also be used to constrain electromagnetic fields in a dS universe. In particular, the backreaction from the Schwinger process can impose severe constraints on cosmological models for generating primordial electromagnetic fields during the inflationary dS phase.

The possibility of the cosmological generation of magnetic fields during the inflationary epoch has been studied by many authors, e.g. [21-27, 42-52], in order to explain the origin of the large-scale magnetic fields in our universe. In such inflationary magnetogenesis scenarios, the magnetic fields are generically accompanied by the production of even larger electric fields. One of the guidelines towards constructing a consistent model has been to keep the production of electric fields under control in order to avoid the electric fields from dominating the energy density of the universe [23-27]. However, we have seen in the previous section that even if the electric fields do not dominate the universe, they can induce large conductivity in the universe via the Schwinger process, which may have nonnegligible backreaction on the Maxwell fields. Therefore, in this section we analyze the effect of the Schwinger process on magnetogenesis in an inflationary dS spacetime. We will 
see that, unless charged fields are absent in the action, or are very massive, the Schwinger effect can spoil the process of magnetogenesis. Through discussing magnetogenesis, we will also see how the induced current backreacts on the background Maxwell fields.

\subsection{Model}

The cosmological enhancement of the electromagnetic fields are realized in inflationary magnetogenesis scenarios by breaking the conformal symmetry of the Maxwell theory. To make our discussions concrete, we focus on a class of models where the conformal symmetry is broken by a time dependent effective coupling $I$ on the Maxwell kinetic term, ${ }^{3}$

$$
S=\int d^{4} x \sqrt{-g}\left\{-\frac{I^{2}}{4} F_{\mu \nu} F^{\mu \nu}-g^{\mu \nu}\left(\partial_{\mu}-i e A_{\mu}\right) \varphi^{*}\left(\partial_{\nu}+i e A_{\nu}\right) \varphi-m^{2} \varphi^{*} \varphi\right\} .
$$

The coupling $I$ can be thought of as a function of other degrees of freedom, such as the inflaton field. As in the previous section, we consider Schwinger process with a charged complex scalar and analyze its effect on inflationary magnetogenesis. ${ }^{4}$

With the effective coupling, the Maxwell equation is modified to

$$
\nabla^{\nu}\left(I^{2} F_{\mu \nu}\right)=J_{\mu}
$$

where the current $J_{\mu}$ is shown in (2.42). In the following, we study the dynamics of the Maxwell fields in the Coulomb gauge, ${ }^{5}$

$$
\partial_{i} A_{i}=A_{\tau}=0
$$

Considering a dS background (2.2), and setting $I$ to be homogeneous, the spatial component of the modified Maxwell equation reads

$$
A_{i}^{\prime \prime}-\partial_{j} \partial_{j} A_{i}+2 \frac{I^{\prime}}{I} A_{i}^{\prime}=\frac{a^{2}}{I^{2}} J_{i}
$$

We discuss electromagnetic fields defined in terms of $A_{\mu}$ (instead of the normalized $\tilde{A}_{\mu}=$ $\left.I A_{\mu}\right)$,

$$
E_{\mu}=u^{\nu} F_{\mu \nu}, \quad B_{\mu}=\frac{1}{2} \varepsilon_{\mu \nu \sigma} F^{\nu \sigma},
$$

because it is $A_{\mu}$ that the complex scalars couple to with charge $e$, and also since we consider the coupling $I$ to be fixed to unity in the present universe. Here $u^{\mu}$ is the 4 -velocity

\footnotetext{
${ }^{3}$ The conformal symmetry can be broken alternatively by a mass term for the photon, $m_{\gamma}^{2} A_{\mu} A^{\mu}$. In such models, significant magnetogenesis requires a tachyonic mass, i.e. $m_{\gamma}^{2}<0$, which for example can arise from non-minimal couplings to gravity [21]. However, such theories have been pointed out to have problems including the appearance of a ghost $[24,53,54]$.

${ }^{4}$ One could also imagine cases where the effective coupling $I$ shows up not only in front of the Maxwell kinetic term, but in front of all the terms in the Lagrangian. In such cases, a time varying $I$ can further induce $\varphi$ production in addition to the Schwinger and gravitational effects, and therefore the backreaction to the Maxwell fields are expected to become stronger, resulting in even more stringent constraints on magnetogenesis than for (3.1).

${ }^{5} A_{\tau}$ cannot be taken to zero in the presence of charge, however, since we only use the equations including $J_{\mu}$ for obtaining a rough criterion for the backreaction from $J_{\mu}$ being non-negligible, we can approximately set $A_{\tau}=0$ in order to simplify the calculations.
} 
of the comoving observer, and $\varepsilon_{\mu \nu \sigma}=\eta_{\mu \nu \sigma \gamma} u^{\gamma}$, where $\eta_{\mu \nu \sigma \gamma}$ is a totally antisymmetric permutation tensor with $\eta_{0123}=-\sqrt{-g}$. Thus $\varepsilon_{i j k}$ is totally antisymmetric with $\varepsilon_{x y z}=a^{3}$. The time components $B_{\tau}$ and $E_{\tau}$ vanish, while the spatial components are

$$
E_{i}=-\frac{1}{a} A_{i}^{\prime}, \quad B_{i}=\frac{1}{a^{4}} \varepsilon_{i j k} \partial_{j} A_{k} .
$$

The magnitude of the fields are

$$
\begin{aligned}
& E^{2} \equiv E_{\mu} E^{\mu}=\frac{1}{a^{2}} E_{i} E_{i}=\frac{1}{a^{4}} A_{i}^{\prime} A_{i}^{\prime}, \\
& B^{2} \equiv B_{\mu} B^{\mu}=\frac{1}{a^{2}} B_{i} B_{i}=\frac{1}{a^{4}}\left(\partial_{i} A_{j} \partial_{i} A_{j}-\partial_{i} A_{j} \partial_{j} A_{i}\right) .
\end{aligned}
$$

Then, with the conductivity

$$
\sigma=\frac{J_{i}}{E_{i}}
$$

(here we do not take the sum over $i$ in the right hand side of (3.9)), the Maxwell equation (3.4) is rewritten as

$$
A_{i}^{\prime \prime}-\partial_{j} \partial_{j} A_{i}+\left(\frac{2 I^{\prime}}{I}+\frac{a \sigma}{I^{2}}\right) A_{i}^{\prime}=0 .
$$

\subsection{In the absence of charged fields}

We shall first discuss the idealized situation where any charged fields are absent in the action. This subsection will also serve as a brief review of magnetogenesis in $I^{2} F F$ scenarios.

Let us focus on large-scale magnetic fields and neglect the spatial derivative term in the Maxwell equation (3.10), giving

$$
A_{i}^{\prime \prime}+\frac{2 I^{\prime}}{I} A_{i}^{\prime}=0
$$

The general solution of this equation is

$$
A_{i}=C_{1}+C_{2} \int \frac{d \tau}{I^{2}}
$$

where $C_{1}$ and $C_{2}$ are constants. For instance, if the coupling $I$ decreases in time as

$$
I \propto a^{-s}, \quad \text { with } \quad s>\frac{1}{2},
$$

then the vector potential possesses a growing mode

$$
A_{i} \propto a^{2 s-1}
$$

and thus the electromagnetic fields are enhanced. Hereafter we suppose $I$ to follow the scaling behavior (3.13) during inflation, and then stays constant after inflation. ${ }^{6}$

\footnotetext{
${ }^{6}$ It could also be that the coupling $I$ approaches a constant at some time $\tau_{1}$ during inflation, and thus magnetogenesis terminates before the end of inflation. In such cases, the constraint on magnetic fields we obtain in (3.29) is modified by $I_{\text {end }} \rightarrow I\left(\tau_{1}\right)$, and also obtains an additional factor of $a\left(\tau_{1}\right) / a_{\text {end }}$ in the right hand side, which makes the bound more stringent.
} 
Focusing on Maxwell fields with a certain wave number $k$, let us rewrite the magnetic field amplitude (3.8) as $^{7}$

$$
B^{2} \sim \frac{k^{2}}{a^{4}} A_{i} A_{i} .
$$

Then, using $A_{i}^{\prime} / A_{i}=(2 s-1) a^{\prime} / a$ which follows from (3.14), the ratio between the electric and magnetic amplitudes with wave number $k$ is obtained as

$$
\left|\frac{E}{B}\right|=(2 s-1) \frac{a H_{\mathrm{inf}}}{k} .
$$

In this section we denote the (nearly) constant Hubble parameter during inflation by the subscript "inf". As can be seen from (3.15), the magnetic field after the magnetogenesis phase decays as $B \propto a^{-2}$. Hence we can obtain a relation between the magnetic field strength in the present universe and the electric field at the end of inflation as

$$
\left|B_{0}\right|=\frac{1}{2 s-1} \frac{k}{a_{0} H_{\text {inf }}} \frac{a_{\text {end }}}{a_{0}}\left|E_{\text {end }}\right| .
$$

Here the subscript "0" denotes quantities in the present epoch, and "end" at the end of inflation. We suppose the post-inflationary universe to be first dominated by an oscillating inflaton, and thus effectively matter-dominated until reheating happens,

$$
\left(\frac{H_{\mathrm{reh}}}{H_{\mathrm{inf}}}\right)^{2}=\left(\frac{a_{\mathrm{end}}}{a_{\mathrm{reh}}}\right)^{3},
$$

where the subscript "reh" denotes quantities at reheating. After reheating, we consider the entropy to be conserved, i.e. $s \propto a^{-3}$, and thus obtain ${ }^{8}$

$$
\frac{a_{\mathrm{reh}}}{a_{0}} \approx 6 \times 10^{-32}\left(\frac{M_{p}}{H_{\mathrm{reh}}}\right)^{1 / 2} .
$$

The combination of (3.18) and (3.20) yields the expansion after inflation,

$$
\frac{a_{\mathrm{end}}}{a_{0}} \approx 6 \times 10^{-32}\left(\frac{H_{\mathrm{reh}}}{H_{\mathrm{inf}}}\right)^{1 / 6}\left(\frac{M_{p}}{H_{\mathrm{inf}}}\right)^{1 / 2},
$$

which allows us to rewrite (3.17) as

$$
\frac{\left|B_{0}\right|}{M_{p}^{2}} \approx \frac{6 \times 10^{-32}}{2 s-1} \frac{k}{a_{0} M_{p}}\left(\frac{H_{\mathrm{reh}}}{H_{\mathrm{inf}}}\right)^{1 / 6}\left(\frac{H_{\mathrm{inf}}}{M_{p}}\right)^{1 / 2} \frac{\left|E_{\mathrm{end}}\right|}{H_{\mathrm{inf}}^{2}} .
$$

\footnotetext{
${ }^{7}$ This approximate expression of $B^{2}$ is good enough for obtaining the $E$ - $B$ ratio (3.16). More detailed derivations of (3.16) can be found in the references.

${ }^{8}$ Upon computing the entropy density at reheating

$$
s_{\mathrm{reh}}=\frac{2 \pi^{2}}{45} g_{s *}\left(T_{\mathrm{reh}}\right)\left(\frac{90}{\pi^{2}} \frac{M_{p}^{2} H_{\mathrm{reh}}^{2}}{g_{*}\left(T_{\mathrm{reh}}\right)}\right)^{3 / 4},
$$

we have chosen the relativistic degrees of freedom to take the maximum value allowed in the MSSM, $g_{*}=g_{s *}=228.75$. However we note that this choice affects (3.20) only by an order unity factor; e.g., $g_{*}=g_{s *}=10.75$ gives a factor 7 instead of 6 in the right hand side.
} 


\subsection{Constraints from Schwinger effect}

Let us now study how the above picture of magnetogenesis is modified under the existence of charged scalars in the action. From the Maxwell equation (3.10), one can read off the criterion for the induced current to be negligible as

$$
\left|\sigma\left(\frac{I^{\prime}}{a I}\right)^{-1}\right| \ll I^{2} .
$$

In other words, when the ratio between $\sigma$ and the rate of magnetogenesis is larger than $\sim I^{2}$, the process of magnetogenesis can be strongly affected by the produced scalars. The $\sigma$ term in (3.10) is seen to be a friction term for $A_{i}^{\prime}$, thus a positive and large $\sigma$ decays away the electric fields and prevents any further magnetogenesis. For the scaling (3.13) under consideration, the criterion (3.23) becomes

$$
\frac{|\sigma|}{H_{\text {inf }}} \ll s I^{2} .
$$

The threshold $I^{2}$ in the right hand sides can be understood from the fact that, when absorbing the coupling by $\tilde{A}_{\mu}=I A_{\mu}$, the effective charge of $\varphi$ becomes $e / I$. Hence a smaller $I$ enhances the backreaction of the produced $\varphi$ on the Maxwell fields. This provides an explicit example of a problem that arises when $I$ is tiny, often referred to in the literature as the strong coupling problem [24, 43]. However, even if $I$ is never smaller than unity, we will see that the criterion (3.24) severely constrains inflationary magnetogenesis.

The criterion (3.24) can be used to set an upper bound on the magnetic fields that can be generated during inflation. We evaluate the bound by using the approximate expression (2.66) for the current under strong electric fields (the validity of using this approximation will shortly be discussed). With the approximation, the conductivity is expressed as

$$
\frac{\sigma}{H_{\mathrm{inf}}} \simeq \frac{1}{12 \pi^{3}} \frac{\left|e^{3} E\right|}{H_{\mathrm{inf}}^{2}} \exp \left(-\frac{\pi m^{2}}{|e E|}\right)
$$

which is solved for the electric field as

$$
\frac{|E|}{H_{\mathrm{inf}}^{2}} \simeq \frac{12 \pi^{3}}{|e|^{3}} \frac{\sigma}{H_{\mathrm{inf}}} \exp \left\{W\left(\frac{e^{2}}{12 \pi^{2}} \frac{m^{2}}{H_{\mathrm{inf}}^{2}} \frac{H_{\mathrm{inf}}}{\sigma}\right)\right\} .
$$

Here, $W(x)$ is the Lambert $W$-function which is the solution of $W e^{W}=x$. For $x \geq 0$, $W(x)$ is a non-negative and increasing function. Thus we note that $|E| / H_{\mathrm{inf}}^{2}$ is an increasing function of $\sigma / H_{\text {inf }}$ when the other parameters are fixed. Moreover, since $W(x) \simeq x$ for $0 \leq x \ll 1$, the exponential factor in (3.26) approaches unity for small $m$. This limit can also be obtained directly by solving the approximation (2.64).

From (3.26), the criterion (3.24) is translated into an upper bound on the electric field during inflation,

$$
\frac{|E|}{H_{\mathrm{inf}}^{2}} \lesssim \frac{12 \pi^{3} s I^{2}}{|e|^{3}} \exp \left\{W\left(\frac{e^{2}}{12 \pi^{2} s I^{2}} \frac{m^{2}}{H_{\mathrm{inf}}^{2}}\right)\right\}
$$


which imposes a bound on the present magnetic field amplitude via (3.22),

$$
\frac{\left|B_{0}\right|}{M_{p}^{2}} \lesssim 10^{-29} \frac{2 s}{2 s-1} \frac{k}{a_{0} M_{p}}\left(\frac{H_{\text {reh }}}{H_{\text {inf }}}\right)^{1 / 6}\left(\frac{H_{\text {inf }}}{M_{p}}\right)^{1 / 2} \frac{I_{\text {end }}^{2}}{|e|^{3}} \exp \left\{W\left(\frac{e^{2}}{12 \pi^{2} s I_{\text {end }}^{2}} \frac{m^{2}}{H_{\text {inf }}^{2}}\right)\right\} .
$$

Here, $I_{\text {end }}$ denotes the value of the effective coupling at the end of inflation. Note from (3.14) that $s$ should be larger than (and not so close to) $1 / 2$ for the Maxwell fields to be significantly enhanced during inflation. Thus $\frac{2 s}{2 s-1}$ should be of order unity for an efficient magnetogenesis. Further noting $H_{\text {reh }} \leq H_{\text {inf }}$, then the bound can be rewritten as

$$
\left|B_{0}\right| \lesssim 10^{-28} \mathrm{G}\left(\frac{k}{a_{0}} \mathrm{Mpc}\right)\left(\frac{H_{\mathrm{inf}}}{M_{p}}\right)^{1 / 2}\left(\frac{\sqrt{4 \pi \alpha}}{|e|}\right)^{3} I_{\text {end }}^{2} \mathcal{Q}
$$

where $\mathcal{Q}$ represents the mass dependence,

$$
\mathcal{Q}=\exp \left\{W\left(\frac{e^{2}}{12 \pi^{2} s I_{\mathrm{end}}^{2}} \frac{m^{2}}{H_{\mathrm{inf}}^{2}}\right)\right\} \simeq \exp \left\{W\left(10^{-3} \frac{e^{2}}{4 \pi \alpha} \frac{1}{s I_{\mathrm{end}}^{2}} \frac{m^{2}}{H_{\mathrm{inf}}^{2}}\right)\right\} .
$$

Here we are using the Heaviside-Lorentz units, thus $1 G \approx 2 \times 10^{-20} \mathrm{GeV}^{2}$, and the elementary charge is $\sqrt{4 \pi \alpha} \approx 0.3$. We also note $1 \mathrm{Mpc} \approx 2 \times 10^{29} \mathrm{eV}^{-1}$. The mass dependent factor $\mathcal{Q}$ is a growing function of $m / H_{\text {inf }}$, with $\lim _{m \rightarrow 0} \mathcal{Q}=1$. Moreover, $\mathcal{Q}$ is of order unity when the argument of $W$ is smaller than $\sim 1$. In other words, the bound (3.29) is independent of the scalar mass if the mass ratio satisfies

$$
\frac{m^{2}}{H_{\mathrm{inf}}^{2}} \lesssim 10^{3} \frac{4 \pi \alpha}{e^{2}} s I_{\text {end }}^{2}
$$

When fixing all the parameters except for $H_{\mathrm{inf}}$, then the magnetic upper bound (3.29) is an increasing function of $H_{\text {inf }}$ while (3.31) is satisfied, scaling as $H_{\text {inf }}^{1 / 2}$. On the other hand, when (3.31) is violated, the $\mathcal{Q}$ factor becomes important and the upper bound turns into a decreasing function of $H_{\text {inf }}$. In figure 2 we plot the upper bound (3.29) as a function of $H_{\text {inf }}$, for a fixed set of parameters $k / a_{0}=1 \mathrm{Mpc}, e^{2}=4 \pi \alpha, m=0.5 \mathrm{MeV}$, and $I_{\text {end }}=1$. The scaling factor $s$ is taken to be of order unity (its explicit value is unimportant here as varying $s$ by order unity makes little difference in the plot). It is clearly seen that the upper bound switches from a decreasing to an increasing function of $H_{\text {inf }}$, as the ratio $m / H_{\text {inf }}$ decreases and starts to satisfy (3.31).

As we have seen, the magnetic field bound (3.29) derive from the Schwinger effect constraint on the electric field (3.27), which can be recast in the form of

$$
\frac{\left|e E_{\text {end }}\right|}{H_{\text {inf }}^{2}} \lesssim 10^{3} \frac{4 \pi \alpha}{e^{2}} s I_{\text {end }}^{2} \mathcal{Q} .
$$

Hence for $e^{2} \sim 4 \pi \alpha$ and $I_{\text {end }} \sim 1$, it is evident that the restrictions on magnetogenesis arise from the Schwinger effect in the large $|\lambda|$ regime, as was assumed upon using the approximation (2.66). Here we remark that a large charge $e^{2} \gg 4 \pi \alpha$ can push the constrained region into the small $|\lambda|$ regime and thus invalidate the usage of (2.66). However, 


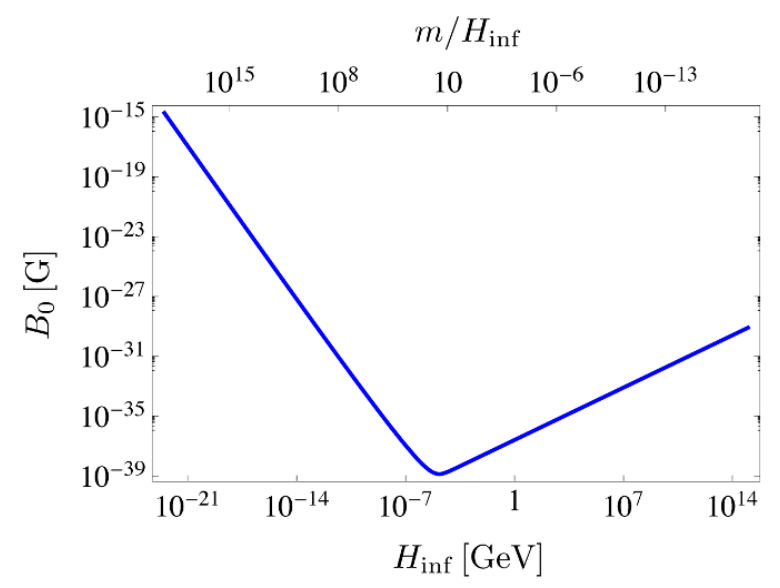

Figure 2. Upper bound (3.29) on the present amplitude of magnetic fields with correlation length $k / a_{0}=1 \mathrm{Mpc}^{-1}$ from the Schwinger effect constraint. A scalar with charge $e^{2}=4 \pi \alpha$ and mass $m=0.5 \mathrm{MeV}$ is assumed. The effective coupling is set to $I_{\text {end }}=1$. The horizontal axes show the inflation scale $H_{\mathrm{inf}}$ (lower) and the ratio $m / H_{\mathrm{inf}}$ (upper). In the region where $m / H_{\text {inf }}$ is tiny, further constraints may arise due to the strong enhancement of the conductivity at the early stage of magnetogenesis (see the main text for details).

the conductivity in the $|\lambda| \ll 1$ regime is generically larger than an extrapolation of (2.66), and thus the bound is expected to be even stronger than the form of (3.29). We also note that the approximation (2.66) is not necessarily valid throughout the $|\lambda| \gg 1$ regime; it is seen through the purple line in figure $1(\mathrm{~b})$ that, as one moves towards smaller $|\lambda|$, the conductivity $\sigma$ eventually deviates from the exponential fall so that it can smoothly connect to the plateau at $|\lambda| \ll 1$. Such behavior also enhances $\sigma$ relative to that predicted by (2.66), and thus gives a magnetic bound stronger than (3.29). The situation becomes more severe for cases with extremely light masses (i.e. $m \ll H_{\text {inf }}$ ), where the conductivity is strongly enhanced at $|\lambda| \ll 1$. The large conductivity under weak electric fields can affect magnetogenesis at its early stage, long before the electromagnetic fields grow to values constrained by the bound (3.29) (though the constraint would also depend on the value of $I$ during inflation). ${ }^{9}$ Therefore the plot in figure 2 should be considered as a conservative bound for regions where the ratio $m / H_{\text {inf }}$ is tiny.

We should also comment on the applicability of the results from the previous section, where we considered a constant and uniform electric field, on inflationary magnetogenesis where the electromagnetic fields with finite correlation lengths are continuously being produced. Here it should be noted that the typical time scale for the enhancement of the electromagnetic fields is, in the case (3.14) under consideration, of order the Hubble time $H^{-1}$. Moreover, the electromagnetic fields are significantly enhanced after exiting the Hubble horizon, and thus we have given constraints on wave modes that are sufficiently

\footnotetext{
${ }^{9}$ We could also say that when $m \ll H_{\text {inf }}$, independently of the magnetogenesis mechanism, the inflationary universe cannot leave behind arbitrarily small electromagnetic fields; the strong enhancement of the conductivity under weak electric fields forbids the electromagnetic fields from existing as a stable background. In this sense, a "lower bound" on the electromagnetic fields exists for inflationary magnetogenesis with light charged particles.
} 
larger than the horizon at the end of the magnetogenesis phase. On the other hand, the constraints are mostly due to the Schwinger process in the $|e E| \gg H_{\mathrm{inf}}^{2}$ regime, and thus the produced charged scalars typically have wave modes much smaller than the Hubble radius, cf. (2.31). In this regime, it could also be checked that the $\varphi$ population is always dominated by the particles newly created within a Hubble time. Thus the length and time scales relevant to the Schwinger process do not exceed those of the electric fields, validating our procedure of modeling the electric fields produced during inflation as being constant and uniform upon evaluating the Schwinger effect constraints.

In figure 3 we plot the upper bound on the amplitude of the magnetic fields in the present universe (3.29) as a function of the correlation length. The charge of the complex scalar is set to the elementary charge $e^{2}=4 \pi \alpha$, and the coupling at the end of inflation to $I_{\text {end }}=1$. The scaling factor $s$ is chosen to be of order unity; the bound depends sufficiently weakly on $s$ such that its explicit value is not important here. For the chosen sets of parameters in the figure, magnetogenesis is constrained by the Schwinger effect in the large $|\lambda|$ regime where the approximation (2.66) is valid, and thus the magnetic bounds can be fully described by the expression (3.29).

Each line represents the upper bound for a different set of the inflation scale $H_{\text {inf }}$ and the scalar mass $m$. The case of a high-scale inflation with $H_{\mathrm{inf}}=10^{14} \mathrm{GeV}$ (corresponding to a tensor-to-scalar ratio of $r \simeq 0.2$, as recently suggested in [55]) is shown as blue lines; the solid line is for $m \lesssim 10 H_{\text {inf }}$, and the dashed line for $m=10^{3} H_{\text {inf }}$. In the former case, the mass dependent factor (3.30) is $\mathcal{Q} \sim 1$, while for the latter $\mathcal{Q} \sim 10^{2}$ and thus the bound is relaxed.

The bounds should be compared with results from recent gamma ray observations, that suggest the existence of intergalactic magnetic fields of strength

$$
\left|B_{0}\right| \gtrsim 10^{-15} \mathrm{G}
$$

when the correlation length is of Mpc scales or larger [28-34]. If the correlation length $\lambda_{B}$ is much smaller than a Mpc, the lower bound improves as $\lambda_{B}^{-1 / 2}$. The suggested magnetic field strength is shown in the figure as the cyan shaded region, where the lower bound is taken from [31] (for the case of extended cascade emission). The observational bound has astrophysical uncertainties (see e.g. $[56,57]$ ) and more detailed work will be required to verify the claim, however we already see from the plot that the Schwinger effect severely constrains the inflationary magnetogenesis scenario from producing such large-scale magnetic fields; the blue solid line shows an upper bound of $\left|B_{0}\right| \lesssim 10^{-30} \mathrm{G}$ on Mpc scale and beyond. For other observational constraints on magnetic fields, see the review [58].

Here we should note that, in order to generate magnetic power of (3.33) on Mpc scales from the magnetogenesis with the scaling behavior (3.13), then the inflation scale should actually satisfy $H_{\mathrm{inf}}<10^{-32} M_{p} \sim 10^{-14} \mathrm{GeV}$, otherwise the produced electric fields end up dominating the energy density of the universe, as derived in [27]. (See also $[23,24,26].)^{10}$

\footnotetext{
${ }^{10}$ Combining (3.22) with the requirement that the electric field should not dominate the energy density of the universe, i.e.,

$$
\rho_{\mathrm{E}} \sim \frac{I^{2}}{2} E^{2}<3 M_{p}^{2} H_{\mathrm{inf}}^{2}
$$




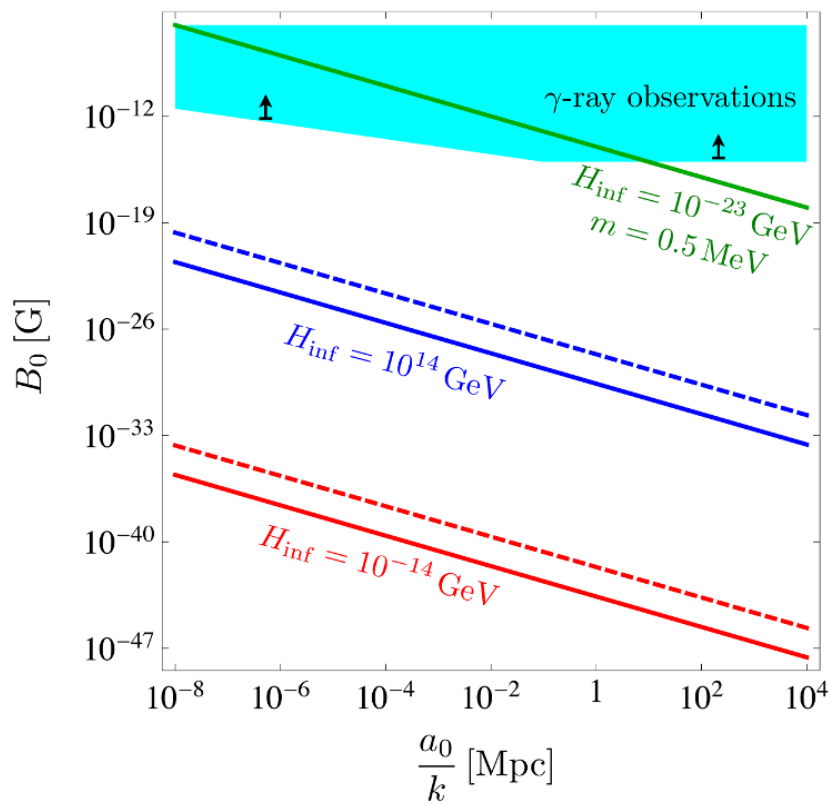

Figure 3. Upper bound on the present amplitude of magnetic fields produced during inflation from Schwinger effect constraints. The blue and red lines are, respectively, for $H_{\text {inf }}=10^{14} \mathrm{GeV}$ and $H_{\text {inf }}=10^{-14} \mathrm{GeV}$, with $m \lesssim 10 H_{\text {inf }}$ (solid lines) and $m=10^{3} H_{\text {inf }}$ (dashed lines). The green line shows the case for the lowest possible inflation scale $H_{\text {inf }}=10^{-23} \mathrm{GeV}$, with $m=0.5 \mathrm{MeV}$. The charge of the complex scalar is fixed to $e^{2}=4 \pi \alpha$, and the effective coupling to $I_{\text {end }}=1$. The cyan shaded region shows the magnitude of intergalactic magnetic fields suggested by gamma-ray observations, whose lower bound is from [31].

Thus in the figure we also plot the magnetic bound for $H_{\mathrm{inf}}=10^{-14} \mathrm{GeV}$ as red lines, again with the solid line for $m \lesssim 10 H_{\text {inf }}$ and the dashed line for $m=10^{3} H_{\text {inf }}$. With such a low inflation scale, the red solid line now gives $\left|B_{0}\right| \lesssim 10^{-44} \mathrm{G}$ on Mpc scales.

As long as $m \lesssim 10 H_{\text {inf }}$, the bound scales as $H_{\text {inf }}^{1 / 2}$ and thus becomes more severe for lower inflation scales. However, the situation is different when the mass is much larger than $H_{\text {inf }}$. We have seen in figure 2 that, when the mass is sufficiently large such that (3.31) is violated, then the upper bound (3.29) turns into a decreasing function of $H_{\text {inf }}$. Thus we further plot the case of a charged field with an electron mass $m=0.5 \mathrm{MeV}$, and an extremely low-scale inflation $H_{\text {inf }}=10^{-23} \mathrm{GeV}$, which is the lowest possible scale compatible with Big Bang Nucleosynthesis [59, 60], although it would require instantaneous reheating and baryogenesis. This extreme case with $m / H_{\text {inf }}=5 \times 10^{19}$ is shown as the green line in the plot. Due to the very large mass-Hubble ratio, the conductivity is suppressed and thus relaxes the bound by $\mathcal{Q} \sim 10^{34}$. In particular, the upper bound on the Mpc scale is $\left|B_{0}\right| \lesssim 10^{-14} \mathrm{G}$, which is comparable to the value of the observational lower bound (3.33).

In summary, under the existence of charged fields in the action, the Schwinger effect introduces a serious obstacle towards inflationary magnetogenesis. This is manifested in the

and further demanding $I_{\text {end }}^{2} \gtrsim 1$ to avoid strong couplings in the theory, one can check that $H_{\text {inf }} \lesssim 10^{-32} M_{p}$ is necessary for magnetic fields of $\left|B_{0}\right| \gtrsim 10^{-15} \mathrm{G}$ to be produced on scales of Mpc or larger. 
form of an upper bound (3.29) on the produced magnetic field, with possible corrections which typically make the bound more severe, as discussed below (3.32). If for example the charged field has a mass of order the Hubble scale or smaller, and carries charge of order the elementary charge, then the Schwinger constraint eliminates the possibility of the discussed inflationary magnetogenesis scenario being responsible for producing the extragalactic magnetic fields (3.33) suggested by gamma ray observations. For cases with extremely light charged fields, i.e. $m \ll H_{\text {inf }}$, the phase of magnetogenesis may not be able to even start, as the induced conductivity under weak electric fields is substantially enhanced. We stress that, as long as the mass of the charged field is not much larger than the Hubble scale, the constraint is more severe for lower inflation scales. Hence lowering the energy scale of inflation (as was considered in $[51,52]$ to circumvent previous constraints) does not improve the situation.

The Schwinger constraint can be relaxed if all charged fields have tiny charges, or if their masses are much larger than the inflationary Hubble scale. For instance, the running of the charge at high energies may suppress the charges during inflation. Alternatively, if inflation is driven by the Higgs field [61], then charged particles in the Standard Model may acquire very large masses while the Higgs field takes large field values. Another possibility of relaxing the constraint is to have a large value for the effective coupling $I$ at the end of the magnetogenesis phase, so that the backreaction from the induced current is suppressed. (We repeat that $I$ here denotes the relative factor between the Maxwell kinetic term and the coupling term between the vector potential and the charged field. Thus when absorbing $I$ into the definition of $A_{\mu}$, then a large $I$ corresponds to a tiny effective charge.) If $I$ is to approach unity in the present universe, then one could imagine a case where $I$ takes a large value at the end of inflation, and keeps decreasing after inflation. Such a possibility was investigated in [27], where magnetogenesis further continues in the post-inflationary epoch until reheating. Constraints from the Schwinger effect can readily be applied for post-inflationary mechanisms of magnetogenesis as well; due to the low Hubble rate, the Schwinger process is expected to receive strong mass suppression, though we defer a careful study of Schwinger effect in post-inflationary scenarios to a future work. We should also note that the Schwinger constraint is evaded if the charged fields are absent from the action during inflation, e.g., it could be that the terms for the charged fields in the action somehow emerge in the post-inflationary universe.

\section{Conclusions}

In this work, we have analyzed particle creation by electric and gravitational fields in a four-dimensional dS space. In addition to the usual Bogoliubov computations, we calculated the expectation value of the induced current. By directly evaluating the current, we could investigate regimes where an adiabatic vacuum does not necessarily exist in the asymptotic future for the charged particles. However, divergences had to be removed from the expectation value of the current operator. To this end, we applied the adiabatic regularization method. We saw that subtracting terms up to quadratic order in the adiabatic 
expansion removes all infinities, while also yields results that have the correct behavior in the flat space limit.

The expression for the regularized current is presented in (2.58). Under strong electric fields $|e E| \gg H^{2}$, the limiting form of the current is shown in (2.63) (or (2.66)), whose behavior coincides with that in flat space. In particular, the linear dependence of the conductivity on the electric field, i.e. $\sigma \propto E$, which is inherent in a four-dimensional space, plays an important role upon constraining electric fields in the early universe. On the other hand, under weak electric fields $|e E| \ll H^{2}$, the approximate expression for the induced current is given in (2.72). The conductivity in this regime is independent of the electric field, and moreover is inversely proportional to the mass squared, i.e. $\sigma \sim 10^{-2} \times e^{2} \mathrm{H} / \mathrm{m}^{2}$. Thus the dS space acquires a large conductivity under weak electric fields for small masses, i.e. $m \ll H$. This intriguing phenomenon with small masses is supported by the infrared modes of the charged scalar, and was also seen to happen in a two-dimensional dS [7]. For massive particles, the contribution to the current from each wave mode does not grow indefinitely towards the infrared, however we note that the scaling $\sigma \propto m^{-2}$ holds for arbitrary mass. Thus even massive charged particles can give rise to a non-negligible conductivity under weak electric fields in four-dimensional dS space. This should be contrasted to the case of two-dimensional dS where $\sigma$ is exponentially suppressed at large masses.

We remark that the exactly massless case cannot be handled in the formalism presented in this paper, as the adiabatic expansion breaks down for the zero modes of the massless field. It should also be noted that loop corrections may generate large masses to the charged scalars, and thus avoid the dS universe from obtaining an extremely large conductivity under weak electric fields. In this paper, we have adopted the method of adiabatic regularization, however it would be important to compute the current with a different regularization or renormalization scheme and compare the results. We leave this for future work.

In the second half of the paper, we applied the above results to the early universe in order to constrain cosmological scenarios for generating large-scale magnetic fields in our universe. We showed that the electric fields generated together with the magnetic fields can induce sufficiently large conductivity to terminate the phase of magnetogenesis. We have especially focused on inflationary magnetogenesis models with a modified Maxwell kinetic term $I(t)^{2} F_{\mu \nu} F^{\mu \mu}$, whose coupling scales as $I \propto a^{-s}$. The main constraints arise from the strong electric field regime $|e E| \gg H^{2}$, where the behavior of the Schwinger process is similar to that in flat space. The upper bound from the Schwinger constraint on the produced magnetic amplitude is given in (3.29), and the bounds at various length scales are displayed in figure 3. For instance, if the charged field has a mass of order the Hubble scale or smaller, and carries charge of order the elementary charge, then magnetic fields with correlation length of $\mathrm{Mpc}$ or larger is bounded as $\left|B_{0}\right| \lesssim 10^{-30} \mathrm{G}$ for all possible inflation scales. Although the explicit bound depends on the masses and charges of the particles, we have shown that unless charged fields are absent from the Lagrangian during inflation, the Schwinger effect makes it a formidable task for inflationary magnetogenesis to produce the extragalactic magnetic fields of $\sim 10^{-15} \mathrm{G}$ suggested by gamma ray observations.

In this paper we have focused on a certain class of inflationary magnetogenesis scenarios, however it would be interesting to systematically constrain inflationary magnetogene- 
sis in general from the Schwinger effect. It is also important to constrain non-inflationary mechanisms, such as the scenario in [27] which generates magnetic fields during the matterdominated phase prior to reheating, and [62-64] during phase transitions.

Through the discussions on magnetogenesis scenarios, we have seen that the Schwinger effect gives rise to strong constraints on electromagnetic fields under the existence of charged fields in the action. This, in turn, suggests the exciting possibility of extracting information about the charged fields in the Lagrangian, from the (non-)detection of primordial magnetic fields in our universe.

\section{Acknowledgments}

We would like to thank Jaume Garriga, Shinji Mukohyama, and Tanmay Vachaspati for useful comments on a draft. TK is also grateful to Marcelo Alvarez, Tony Chu, Chris Thompson, Yuki Watanabe, and Aaron Zimmerman for very helpful discussions. This work was supported by the Natural Science and Engineering Research Council of Canada, the University of Waterloo and Perimeter Institute for Theoretical Physics. Research at the Perimeter Institute is supported by the Government of Canada through Industry Canada and by the Province of Ontario through the Ministry of Research \& Innovation.

\section{A Some properties of Whittaker functions}

In this appendix we lay out some of the properties of the Whittaker Functions that are useful for the discussions in section 2. For more details, see e.g. [65].

The Whittaker functions

$$
\begin{aligned}
& W_{\kappa, \mu}(z)=e^{-\frac{z}{2}} z^{\frac{1}{2}+\mu} U\left(\frac{1}{2}+\mu-\kappa, 1+2 \mu, z\right), \\
& M_{\kappa, \mu}(z)=e^{-\frac{z}{2}} z^{\frac{1}{2}+\mu} M\left(\frac{1}{2}+\mu-\kappa, 1+2 \mu, z\right),
\end{aligned}
$$

defined in terms of Kummer's confluent hypergeometric functions $U$ and $M$, are solutions of the differential equation

$$
\frac{d^{2} \mathcal{W}}{d z^{2}}+\left\{\frac{1}{z^{2}}\left(\frac{1}{4}-\mu^{2}\right)+\frac{\kappa}{z}-\frac{1}{4}\right\} \mathcal{W}=0
$$

Here, $M_{\kappa, \mu}(z)$ does not exist when $2 \mu=-1,-2, \cdots$. The fundamental pairs of solutions of (A.2) are formed by $W_{\kappa, \mu}(z), W_{-\kappa, \mu}\left(e^{\pi i} z\right)$ (for $\left.-\frac{3}{2} \pi \leq \arg z \leq \frac{1}{2} \pi\right)$, or $M_{\kappa, \mu}(z), M_{\kappa,-\mu}(z)$ (for $-\pi \leq \arg z \leq \pi$ and $2 \mu \neq 0, \pm 1, \pm 2, \cdots$ ).

The functions have the properties

$$
\begin{aligned}
\left(W_{\kappa, \mu}(z)\right)^{*} & =W_{\kappa^{*}, \mu^{*}}\left(z^{*}\right), & \left(M_{\kappa, \mu}(z)\right)^{*} & =M_{\kappa^{*}, \mu^{*}}\left(z^{*}\right), \\
W_{\kappa, \mu}(z) & =W_{\kappa,-\mu}(z), & M_{\kappa, \mu}\left(z e^{ \pm \pi i}\right) & = \pm i e^{ \pm \mu \pi i} M_{-\kappa, \mu}(z),
\end{aligned}
$$

and are related through the formula (for $2 \mu \neq 0, \pm 1, \pm 2, \cdots$ ):

$$
W_{\kappa, \mu}(z)=\frac{\Gamma(-2 \mu)}{\Gamma\left(\frac{1}{2}-\mu-\kappa\right)} M_{\kappa, \mu}(z)+\frac{\Gamma(2 \mu)}{\Gamma\left(\frac{1}{2}+\mu-\kappa\right)} M_{\kappa,-\mu}(z) .
$$


The Wronskians are

$$
\begin{aligned}
W_{\kappa, \mu}(z) \frac{d W_{-\kappa, \mu}\left(e^{ \pm \pi i} z\right)}{d z}-\frac{d W_{\kappa, \mu}(z)}{d z} W_{-\kappa, \mu}\left(e^{ \pm \pi i} z\right) & =e^{\mp \kappa \pi i}, \\
M_{\kappa, \mu}(z) \frac{d M_{\kappa,-\mu}(z)}{d z}-\frac{d M_{\kappa, \mu}(z)}{d z} M_{\kappa,-\mu}(z) & =-2 \mu .
\end{aligned}
$$

As $|z| \rightarrow \infty$, the function $W_{\kappa, \mu}(z)$ has a limiting form of

$$
W_{\kappa, \mu}(z) \sim e^{-z / 2} z^{\kappa}, \quad \text { for }|\arg z|<\frac{3}{2} \pi .
$$

As $z \rightarrow 0$, the function $M_{\kappa, \mu}(z)$ approaches

$$
M_{\kappa, \mu}(z) \sim z^{\mu+1 / 2} .
$$

We note that, in this paper we take the principal values $-\pi \leq \arg \varpi \leq \pi$ for the phase of complex numbers $\varpi$.

\section{B Computation of the current before regularization}

In this appendix we perform the three-dimensional momentum integral in (2.44) for obtaining the expectation value of the current before its divergences are regularized. We follow the calculational procedure in [7] for a one-dimensional momentum integral, with some modifications along the way.

The integral under consideration is

$$
\begin{aligned}
\int d^{3} k\left(k_{z}+e A_{z}\right) \frac{e^{i \kappa \pi}}{2 k}\left|W_{\kappa, \mu}(z)\right|^{2} & =2 \pi \int_{0}^{\infty} d k k^{2} \int_{-1}^{1} d r\left(k r+\frac{\lambda}{\tau}\right) \frac{e^{r \lambda \pi}}{2 k}\left|W_{-i r \lambda, \mu}(2 k i \tau)\right|^{2} \\
& =-\frac{\pi}{\tau^{3}} \lim _{\xi \rightarrow \infty} \int_{0}^{\xi} d v v \int_{-1}^{1} d r(r v-\lambda) e^{r \lambda \pi}\left|W_{-i r \lambda, \mu}(-2 i v)\right|^{2},
\end{aligned}
$$

where we introduced real variables:

$$
r=\frac{k_{z}}{k}, \quad \lambda=\frac{e E}{H^{2}}, \quad v=-k \tau .
$$

We have also put a cutoff $\xi$ on the $v$-integral, which we will take to infinity at the end of the calculation. Note from the definition (2.18) that $\mu$ is either real or purely imaginary, and that its real part lies in the range of $0 \leq|\operatorname{Re}(\mu)| \leq \frac{3}{2}$. In the following analyses we further suppose

$$
\mu^{2} \neq 0, \frac{1}{4}, 1, \frac{9}{4}
$$

for calculational convenience. The excluded cases can be approached by taking the limits $\mu^{2} \rightarrow 0, \frac{1}{4}, 1, \frac{9}{4}$ of the final result.

Let us rewrite the Whittaker function using the Mellin-Barnes integral representation that is valid for $\frac{1}{2} \pm \mu-\kappa \neq 0,-1,-2, \ldots$, and $|\arg z|<\frac{3}{2} \pi$ (recall that in this paper we take the principal values $-\pi \leq \arg \varpi \leq \pi$ for the phase of complex numbers):

$$
W_{\kappa, \mu}(z)=\frac{e^{-z / 2}}{2 \pi i} \int_{-i \infty}^{i \infty} \frac{\Gamma\left(\frac{1}{2}+\mu+s\right) \Gamma\left(\frac{1}{2}-\mu+s\right) \Gamma(-\kappa-s)}{\Gamma\left(\frac{1}{2}+\mu-\kappa\right) \Gamma\left(\frac{1}{2}-\mu-\kappa\right)} z^{-s} d s,
$$


where the contour of integration separates the poles of $\Gamma\left(\frac{1}{2}+\mu+s\right) \Gamma\left(\frac{1}{2}-\mu+s\right)$ from those of $\Gamma(-\kappa-s)$. Also using $\left(W_{-i r \lambda, \mu}(-2 i v)\right)^{*}=W_{i r \lambda, \mu^{*}}(2 i v)$, then (B.1) can be rewritten as

$$
\begin{aligned}
-\frac{\pi}{\tau^{3}} & \lim _{\xi \rightarrow \infty} \int_{0}^{\xi} d v v \int_{-1}^{1} d r(r v-\lambda) e^{r \lambda \pi} \\
& \times \int_{-i \infty}^{i \infty} \frac{d s}{2 \pi i} \frac{\Gamma\left(\frac{1}{2}+\mu+s\right) \Gamma\left(\frac{1}{2}-\mu+s\right) \Gamma(i r \lambda-s)}{\Gamma\left(\frac{1}{2}+\mu+i r \lambda\right) \Gamma\left(\frac{1}{2}-\mu+i r \lambda\right)}(-2 i v)^{-s} \\
& \times \int_{-i \infty}^{i \infty} \frac{d t}{2 \pi i} \frac{\Gamma\left(\frac{1}{2}+\mu^{*}+t\right) \Gamma\left(\frac{1}{2}-\mu^{*}+t\right) \Gamma(-i r \lambda-t)}{\Gamma\left(\frac{1}{2}+\mu^{*}-i r \lambda\right) \Gamma\left(\frac{1}{2}-\mu^{*}-i r \lambda\right)}(2 i v)^{-t} .
\end{aligned}
$$

Note that the $\mu^{*}$ 's in the expression can be converted into $\mu$ 's, since $\mu^{*}$ is equal to either $\mu$ or $-\mu$.

The integration contours of $s$ and $t$ are arbitrary, as long as they separate the poles as discussed below (B.4), and run from minus to plus infinity in the imaginary direction. Therefore, we choose the contours to always satisfy

$$
\operatorname{Re}(s), \operatorname{Re}(t)<1
$$

Then the $v$-integral in (B.5) can be carried out as

$$
\begin{aligned}
-\frac{\pi}{\tau^{3}} \lim _{\xi \rightarrow \infty} & \int_{-1}^{1} d r \frac{e^{r \lambda \pi}}{\Gamma\left(\frac{1}{2}+\mu+i r \lambda\right) \Gamma\left(\frac{1}{2}-\mu+i r \lambda\right) \Gamma\left(\frac{1}{2}+\mu-i r \lambda\right) \Gamma\left(\frac{1}{2}-\mu-i r \lambda\right)} \\
& \times \int_{-i \infty}^{i \infty} \frac{d s}{2 \pi i} \Gamma\left(\frac{1}{2}+\mu+s\right) \Gamma\left(\frac{1}{2}-\mu+s\right) \Gamma(i r \lambda-s) \int_{-i \infty}^{i \infty} \frac{d t}{2 \pi i} f_{r, s}(t),
\end{aligned}
$$

where

$$
\begin{aligned}
f_{r, s}(t)= & \Gamma\left(\frac{1}{2}+\mu+t\right) \Gamma\left(\frac{1}{2}-\mu+t\right) \Gamma(-i r \lambda-t) \\
& \times \frac{1}{4} e^{i \frac{\pi}{2}(s-t)}(2 \xi)^{2-s-t}\left(\frac{r \xi}{3-s-t}-\frac{\lambda}{2-s-t}\right) .
\end{aligned}
$$

For a fixed set of $r$ and $s$, the function $f_{r, s}(t)$ can have singularities at $t=-\frac{1}{2} \pm \mu-n$ (where $n=0,1,2, \cdots$ ), located on the left side of the integration contour of $t$, and $t=$ $-i r \lambda+n, 2-s, 3-s$, on the right side of the contour.

Upon integrating $f_{r, s}(t)$ over $t$, let us further specify the integration contour of $s$ by requiring

$$
-1<\operatorname{Re}(s)
$$

to be always satisfied. We then carry out the $t$-integral by closing its contour in the right half-plane, without passing through any of the poles. The added integration contour of $t$ does not contribute to the result, since an integral of $f_{r, s}(t)$ over a finite path along the real direction vanishes at $\operatorname{Im}(t) \rightarrow \pm \infty$, and also because any integral in the region $\operatorname{Re}(t)>4$ vanishes in the limit $\xi \rightarrow \infty$ due to the condition (B.9). The residues of $f_{r, s}(t)$ inside the closed contour also vanish as $\xi \rightarrow \infty$, except for those at the simple poles:

$$
t=-i r \lambda,-i r \lambda+1,-i r \lambda+2,-i r \lambda+3,2-s, 3-s
$$


Among the six poles, the ones at $t=-i r \lambda, \cdots,-i r \lambda+3$ give residues that have explicit $\xi$-dependence, while the residues at $t=2-s, 3-s$ are independent of $\xi$. Instead of showing the full expression, in order to reduce clutter we schematically write

$$
\lim _{\xi \rightarrow \infty} \int \frac{d t}{2 \pi i} f_{r, s}(t)=\lim _{\xi \rightarrow \infty} \mathcal{O}\left(\xi^{-s+i r \lambda+3}, \ldots, \xi^{-s+i r \lambda}\right)+\mathcal{O}\left(\xi^{0}\right) .
$$

The $s$-integral in (B.7) with the $\xi$-dependent terms in (B.11) can be carried out similarly to the $t$-integral; closing the contour in the right half-plane, the only residues that survive as $\xi \rightarrow \infty$ are those at

$$
s=i r \lambda, i r \lambda+1, i r \lambda+2, i r \lambda+3 .
$$

These poles are not necessarily simple poles, and thus gives an expression that involves digamma functions $\psi(z)=\Gamma^{\prime}(z) / \Gamma(z)$,

$$
\begin{gathered}
\lim _{\xi \rightarrow \infty} \int_{-i \infty}^{i \infty} \frac{d s}{2 \pi i} \Gamma\left(\frac{1}{2}+\mu+s\right) \Gamma\left(\frac{1}{2}-\mu+s\right) \Gamma(i r \lambda-s) \mathcal{O}\left(\xi^{-s+i r \lambda+3}, \ldots, \xi^{-s+i r \lambda}\right) \\
=e^{-r \lambda \pi} \Gamma\left(\frac{1}{2}+\mu+i r \lambda\right) \Gamma\left(\frac{1}{2}-\mu+i r \lambda\right) \Gamma\left(\frac{1}{2}+\mu-i r \lambda\right) \Gamma\left(\frac{1}{2}-\mu-i r \lambda\right) \\
\times \lim _{\xi \rightarrow \infty}\left[\frac{r}{3} \xi^{3}-\frac{\lambda}{2}\left(1-r^{2}\right) \xi^{2}-\frac{r}{8}\left\{1-4 \mu^{2}+4\left(2-3 r^{2}\right) \lambda^{2}\right\} \xi\right. \\
+\frac{\lambda}{8}\left\{1-4 \mu^{2}+\left(-7-12 \lambda^{2}+12 \mu^{2}\right) r^{2}+20 \lambda^{2} r^{4}\right\} \\
\left.\times\left\{\ln (2 \xi)-\psi\left(\frac{1}{2}+i r \lambda-\mu\right)-\psi\left(\frac{1}{2}+i r \lambda+\mu\right)\right\}+\cdots\right] .
\end{gathered}
$$

In the final line, we have abbreviated terms that are independent of $\xi$ by dots.

On the other hand, the $s$-integral of the $\xi$-independent terms in (B.11) can be written as,

$$
\begin{aligned}
& \int_{-i \infty}^{i \infty} \frac{d s}{2 \pi i} \Gamma\left(\frac{1}{2}+\mu+s\right) \Gamma\left(\frac{1}{2}-\mu+s\right) \Gamma(i r \lambda-s) \mathcal{O}\left(\xi^{0}\right) \\
& =\int_{-i \infty}^{i \infty} \frac{d s}{2 \pi i} \frac{e^{i \pi s}}{\cos \{\pi(\mu+s)\} \cos \{\pi(-\mu+s)\} \sin \{\pi(s-i r \lambda)\}} \\
& \quad \times\left\{\frac{d_{r}}{s-i r \lambda}+g_{r}(s)-g_{r}(s-1)\right\} .
\end{aligned}
$$

Here, $g_{r}(s)$ is a function of the form

$$
g_{r}(s)=\frac{c_{r,-2}}{s-i r \lambda-2}+\frac{c_{r,-1}}{s-i r \lambda-1}+\frac{c_{r, 0}}{s-i r \lambda}+c_{r, 1} s+c_{r, 2} s^{2}+c_{r, 3} s^{3},
$$

and $d_{r}, c_{r,-2}, \ldots, c_{r, 3}$ are independent of $s$. In order to integrate the term with $d_{r}$, let us temporarily consider integrating the modified function

$$
F_{p}(s)=\frac{e^{i \pi s}}{\cos \{\pi(\mu+s)\} \cos \{\pi(-\mu+s)\} \sin \{\pi(s-i r \lambda)\}} \frac{1}{(s-i r \lambda)^{p}},
$$


where the power $p$ is a constant that satisfies $p>1$. Closing the contour path of $s$ on the left half-plane with a semicircle of infinite radius (here consider an arc that does not pass through any of the poles, and taking the infinite radius limit in a discontinuous manner), one can check that the integral of $F_{p}(s)$ along the arc vanishes due to $p>1$.

Here, in addition to the requirements for the integration contour of $s$ explained below (B.4), and at (B.6), (B.9), we further demand the contour to pass to the left of $s=\frac{1}{2} \pm \mu, \frac{3}{2} \pm \mu$. Such a path always exists for $\mu$ satisfying (B.3). Then the integral of $F_{p}(s)$ can be obtained by summing up its infinite series of residues at the simple poles:

$$
s=-\frac{1}{2} \pm \mu-n, \text { ir } \lambda-1-n,
$$

where $n=0,1,2, \ldots$ Then we take the limit $p \rightarrow 1$ of the integrated result in order to obtain the integral of the $d_{r}$ term in (B.14), which can be checked to take the form

$$
\begin{aligned}
\lim _{p \rightarrow 1} \int_{-i \infty}^{i \infty} \frac{d s}{2 \pi i} F_{p}(s)= & -\frac{\gamma e^{-\pi r \lambda}}{\pi \cos \{\pi(\mu+i r \lambda)\} \cos \{\pi(\mu-i r \lambda)\}} \\
& -\frac{i}{\pi \sin (2 \pi \mu)}\left[\frac{e^{-i \pi \mu} \psi\left(\frac{1}{2}+\mu+i r \lambda\right)}{\cos \{\pi(\mu+i r \lambda)\}}-\frac{e^{i \pi \mu} \psi\left(\frac{1}{2}-\mu+i r \lambda\right)}{\cos \{\pi(\mu-i r \lambda)\}}\right] .
\end{aligned}
$$

Here, $\gamma$ is Euler's constant.

As for integrating $g_{r}(s)-g_{r}(s-1)$ in (B.14), we shift the variable for $g_{r}(s-1)$ by $s \rightarrow s+1$, giving

$$
\left(\int_{-i \infty}^{i \infty}-\int_{-i \infty-1}^{i \infty-1}\right) \frac{d s}{2 \pi i} \frac{e^{i \pi s} g_{r}(s)}{\cos \{\pi(\mu+s)\} \cos \{\pi(-\mu+s)\} \sin \{\pi(s-i r \lambda)\}} .
$$

This can be evaluated by computing the residues of poles in the region sandwiched by the original integration contour and the shifted one (the choice of the contour was discussed above (B.17)), which are simple poles at

$$
s=i r \lambda-1,-\frac{1}{2} \pm \mu .
$$

Summing up the contributions from (B.13), (B.18), (B.19), and integrating over $r$, we arrive at the final result:

$$
\begin{aligned}
& \int d^{3} k\left(k_{z}+e A_{z}\right) \frac{e^{i \kappa \pi}}{2 k}\left|W_{\kappa, \mu}(z)\right|^{2} \\
=\frac{\pi}{\tau^{3}} \lim _{\xi \rightarrow \infty}\left[\frac{2 \lambda}{3} \xi^{2}+\frac{\lambda}{3} \ln (2 \xi)-\frac{25 \lambda}{36}-\frac{i \pi \lambda}{6}+\frac{\mu^{2} \lambda}{3}+\frac{\lambda^{3}}{15}\right. & \\
+ & \frac{45+4 \pi^{2}\left(-2+3 \lambda^{2}+2 \mu^{2}\right)}{12 \pi^{3}} \frac{\mu \cosh (2 \pi \lambda)}{\lambda \sin (2 \pi \mu)}-\frac{45+8 \pi^{2}\left(-1+9 \lambda^{2}+\mu^{2}\right)}{24 \pi^{4}} \frac{\mu \sinh (2 \pi \lambda)}{\lambda^{2} \sin (2 \pi \mu)} \\
+ & \int_{-1}^{1} d r \frac{i \lambda}{16 \sin (2 \pi \mu)}\left\{-1+4 \mu^{2}+\left(7+12 \lambda^{2}-12 \mu^{2}\right) r^{2}-20 \lambda^{2} r^{4}\right\} \\
& \left.\times\left\{\left(e^{2 \pi r \lambda}+e^{2 \pi i \mu}\right) \psi\left(\frac{1}{2}+\mu+i r \lambda\right)-\left(e^{2 \pi r \lambda}+e^{-2 \pi i \mu}\right) \psi\left(\frac{1}{2}-\mu+i r \lambda\right)\right\}\right] .
\end{aligned}
$$


We see that the three-dimensional momentum integral for the current has quadratic and logarithmic divergences. (The cutoff $\xi$ is related to that in (2.46) by $\xi=-\tau \zeta$.) Let us also note that the integral for the values of $\mu$ excluded in (B.3) can be evaluated by taking the limits of the result (B.21). It can further be checked that the imaginary term $-\frac{i \pi \lambda}{6}$ in the second line cancels with the imaginary part of the $r$-integral in the forth and fifth lines, making it evident that the result is real. Thus, with a normalization factor, we obtain the expression (2.46).

Open Access. This article is distributed under the terms of the Creative Commons Attribution License (CC-BY 4.0), which permits any use, distribution and reproduction in any medium, provided the original author(s) and source are credited.

\section{References}

[1] F. Sauter, Uber das Verhalten eines Elektrons im homogenen elektrischen Feld nach der relativistischen Theorie Diracs, Z. Phys. 69 (1931) 742 [INSPIRE].

[2] W. Heisenberg and H. Euler, Consequences of Dirac's theory of positrons, Z. Phys. 98 (1936) 714 [physics/0605038] [INSPIRE].

[3] J.S. Schwinger, On gauge invariance and vacuum polarization, Phys. Rev. 82 (1951) 664 [INSPIRE].

[4] A.I. Nikishov, Barrier scattering in field theory removal of klein paradox, Nucl. Phys. B 21 (1970) 346 [INSPIRE].

[5] N.B. Narozhnyi and A.I. Nikishov, The Simplist processes in the pair creating electric field, Yad. Fiz. 11 (1970) 1072 [INSPIRE].

[6] J. Martin, Inflationary perturbations: The Cosmological Schwinger effect, Lect. Notes Phys. 738 (2008) 193 [arXiv:0704.3540] [INSPIRE].

[7] M.B. Fröb, J. Garriga, S. Kanno, M. Sasaki, J. Soda et al., Schwinger effect in de Sitter space, JCAP 04 (2014) 009 [arXiv: 1401.4137] [INSPIRE].

[8] J. Garriga, Pair production by an electric field in (1+1)-dimensional de Sitter space, Phys. Rev. D 49 (1994) 6343 [INSPIRE].

[9] F. Cooper and E. Mottola, Quantum Back Reaction in Scalar QED as an Initial Value Problem, Phys. Rev. D 40 (1989) 456 [inSPIRE].

[10] Y. Kluger, J.M. Eisenberg, B. Svetitsky, F. Cooper and E. Mottola, Pair production in a strong electric field, Phys. Rev. Lett. 67 (1991) 2427 [INSPIRE].

[11] Y. Kluger, J.M. Eisenberg, B. Svetitsky, F. Cooper and E. Mottola, Fermion pair production in a strong electric field, Phys. Rev. D 45 (1992) 4659 [InSPIRE].

[12] F. Cooper, J.M. Eisenberg, Y. Kluger, E. Mottola and B. Svetitsky, Particle production in the central rapidity region, Phys. Rev. D 48 (1993) 190 [hep-ph/9212206] [INSPIRE].

[13] P.R. Anderson and E. Mottola, On the Instability of Global de Sitter Space to Particle Creation, Phys. Rev. D 89 (2014) 104038 [arXiv:1310.0030] [InSPIRE].

[14] P.R. Anderson and E. Mottola, Quantum Vacuum Instability of 'Eternal' de Sitter Space, Phys. Rev. D 89 (2014) 104039 [arXiv:1310.1963] [INSPIRE]. 
[15] L. Parker and S.A. Fulling, Adiabatic regularization of the energy momentum tensor of a quantized field in homogeneous spaces, Phys. Rev. D 9 (1974) 341 [INSPIRE].

[16] S.A. Fulling and L. Parker, Renormalization in the theory of a quantized scalar field interacting with a robertson-walker spacetime, Annals Phys. 87 (1974) 176 [INSPIRE].

[17] S.A. Fulling, L. Parker and B.L. Hu, Conformal energy-momentum tensor in curved spacetime: Adiabatic regularization and renormalization, Phys. Rev. D 10 (1974) 3905 [INSPIRE].

[18] T.S. Bunch, Calculation of the Renormalized Quantum Stress Tensor by Adiabatic Regularization in Two-Dimensional and Four-Dimensional Robertson-Walker Space-Times, J. Phys. A 11 (1978) 603 [inSPIRE].

[19] T.S. Bunch, Adiabatic regularization for scalar fields with arbitrary coupling to the scalar curvature, J. Phys. A 13 (1980) 1297 [inSPIRE].

[20] P.R. Anderson and L. Parker, Adiabatic Regularization in Closed Robertson-walker Universes, Phys. Rev. D 36 (1987) 2963 [INSPIRE].

[21] M.S. Turner and L.M. Widrow, Inflation Produced, Large Scale Magnetic Fields, Phys. Rev. D 37 (1988) 2743 [INSPIRE].

[22] B. Ratra, Cosmological 'seed' magnetic field from inflation, Astrophys. J. 391 (1992) L1 [INSPIRE].

[23] K. Bamba and J. Yokoyama, Large scale magnetic fields from inflation in dilaton electromagnetism, Phys. Rev. D 69 (2004) 043507 [astro-ph/0310824] [INSPIRE].

[24] V. Demozzi, V. Mukhanov and H. Rubinstein, Magnetic fields from inflation?, JCAP 08 (2009) 025 [arXiv:0907.1030] [INSPIRE].

[25] S. Kanno, J. Soda and M.-a. Watanabe, Cosmological Magnetic Fields from Inflation and Backreaction, JCAP 12 (2009) 009 [arXiv:0908.3509] [INSPIRE].

[26] T. Fujita and S. Mukohyama, Universal upper limit on inflation energy scale from cosmic magnetic field, JCAP 10 (2012) 034 [arXiv:1205.5031] [INSPIRE].

[27] T. Kobayashi, Primordial Magnetic Fields from the Post-Inflationary Universe, JCAP 05 (2014) 040 [arXiv:1403.5168] [INSPIRE].

[28] F. Tavecchio, G. Ghisellini, L. Foschini, G. Bonnoli, G. Ghirlanda et al., The intergalactic magnetic field constrained by Fermi/LAT observations of the TeV blazar 1ES 0229+200, Mon. Not. Roy. Astron. Soc. 406 (2010) L70 [arXiv:1004.1329] [InSPIRE].

[29] A. Neronov and I. Vovk, Evidence for strong extragalactic magnetic fields from Fermi observations of TeV blazars, Science 328 (2010) 73 [arXiv:1006.3504] [INSPIRE].

[30] S. Ando and A. Kusenko, Evidence for Gamma-Ray Halos Around Active Galactic Nuclei and the First Measurement of Intergalactic Magnetic Fields, Astrophys. J. 722 (2010) L39 [arXiv: 1005.1924] [INSPIRE].

[31] A.M. Taylor, I. Vovk and A. Neronov, Extragalactic magnetic fields constraints from simultaneous GeV-TeV observations of blazars, Astron. Astrophys. 529 (2011) A144 [arXiv: 1101.0932] [INSPIRE].

[32] K. Takahashi, M. Mori, K. Ichiki, S. Inoue and H. Takami, Lower Bounds on Magnetic Fields in Intergalactic Voids from Long-term GeV-TeV Light Curves of the Blazar Mrk 421, Astrophys. J. 771 (2013) L42 [INSPIRE]. 
[33] Fermi-LAT collaboration, J. Finke, L. Reyes and M. Georganopoulos, Constraints on the Intergalactic Magnetic Field from Gamma-Ray Observations of Blazars, eConf C $\mathbf{1 2 1 0 2 8}$ (2012) 365 [arXiv:1303.5093] [INSPIRE].

[34] H. Tashiro, W. Chen, F. Ferrer and T. Vachaspati, Search for CP-violating Signature of Intergalactic Magnetic Helicity in the Gamma Ray Sky, arXiv:1310.4826 [INSPIRE].

[35] E.D. Stewart, Inflation, supergravity and superstrings, Phys. Rev. D 51 (1995) 6847 [hep-ph/9405389] [INSPIRE].

[36] M. Dine, L. Randall and S.D. Thomas, Supersymmetry breaking in the early universe, Phys. Rev. Lett. 75 (1995) 398 [hep-ph/9503303] [INSPIRE].

[37] A.A. Starobinsky and J. Yokoyama, Equilibrium state of a selfinteracting scalar field in the de Sitter background, Phys. Rev. D 50 (1994) 6357 [astro-ph/9407016] [InSPIRE].

[38] R.P. Woodard, Generalizing Starobinskii's Formalism to Yukawa Theory 8 to Scalar QED, J. Phys. Conf. Ser. 68 (2007) 012032 [gr-qc/0608037] [InSPIRE].

[39] C.P. Burgess, R. Holman, L. Leblond and S. Shandera, Breakdown of Semiclassical Methods in de Sitter Space, JCAP 10 (2010) 017 [arXiv:1005.3551] [INSPIRE].

[40] J. Serreau, Effective potential for quantum scalar fields on a de Sitter geometry, Phys. Rev. Lett. 107 (2011) 191103 [arXiv:1105.4539] [INSPIRE].

[41] D. Boyanovsky, Condensates and quasiparticles in inflationary cosmology: mass generation and decay widths, Phys. Rev. D 85 (2012) 123525 [arXiv: 1203.3903] [InSPIRE].

[42] W.D. Garretson, G.B. Field and S.M. Carroll, Primordial magnetic fields from pseudoGoldstone bosons, Phys. Rev. D 46 (1992) 5346 [hep-ph/9209238] [InSPIRE].

[43] M. Gasperini, M. Giovannini and G. Veneziano, Primordial magnetic fields from string cosmology, Phys. Rev. Lett. 75 (1995) 3796 [hep-th/9504083] [INSPIRE].

[44] M. Giovannini and M.E. Shaposhnikov, Primordial magnetic fields from inflation?, Phys. Rev. D 62 (2000) 103512 [hep-ph/0004269] [INSPIRE].

[45] A.-C. Davis, K. Dimopoulos, T. Prokopec and O. Tornkvist, Primordial spectrum of gauge fields from inflation, Phys. Lett. B 501 (2001) 165 [astro-ph/0007214] [INSPIRE].

[46] M.M. Anber and L. Sorbo, N-flationary magnetic fields, JCAP 10 (2006) 018 [astro-ph/0606534] [INSPIRE].

[47] J. Martin and J. Yokoyama, Generation of Large-Scale Magnetic Fields in Single-Field Inflation, JCAP 01 (2008) 025 [arXiv:0711.4307] [INSPIRE].

[48] K. Bamba and S.D. Odintsov, Inflation and late-time cosmic acceleration in non-minimal Maxwell-F $(R)$ gravity and the generation of large-scale magnetic fields, JCAP 04 (2008) 024 [arXiv:0801.0954] [INSPIRE].

[49] R. Emami, H. Firouzjahi and M.S. Movahed, Inflation from Charged Scalar and Primordial Magnetic Fields?, Phys. Rev. D 81 (2010) 083526 [arXiv:0908.4161] [INSPIRE].

[50] R. Durrer, L. Hollenstein and R.K. Jain, Can slow roll inflation induce relevant helical magnetic fields?, JCAP 03 (2011) 037 [arXiv: 1005.5322] [INSPIRE].

[51] R.J.Z. Ferreira, R.K. Jain and M.S. Sloth, Inflationary magnetogenesis without the strong coupling problem, JCAP 10 (2013) 004 [arXiv:1305.7151] [INSPIRE]. 
[52] R.J.Z. Ferreira, R.K. Jain and M.S. Sloth, Inflationary Magnetogenesis without the Strong Coupling Problem II: Constraints from CMB anisotropies and B-modes, JCAP 06 (2014) 053 [arXiv: 1403.5516] [INSPIRE].

[53] G. Dvali, O. Pujolàs and M. Redi, Consistent Lorentz Violation in Flat and Curved Space, Phys. Rev. D 76 (2007) 044028 [hep-th/0702117] [INSPIRE].

[54] B. Himmetoglu, C.R. Contaldi and M. Peloso, Ghost instabilities of cosmological models with vector fields nonminimally coupled to the curvature, Phys. Rev. D 80 (2009) 123530 [arXiv:0909.3524] [INSPIRE].

[55] BICEP2 collaboration, P.A.R. Ade et al., Detection of B-Mode Polarization at Degree Angular Scales by BICEPQ, Phys. Rev. Lett. 112 (2014) 241101 [arXiv:1403.3985] [INSPIRE].

[56] A.E. Broderick, P. Chang and C. Pfrommer, The Cosmological Impact of Luminous TeV Blazars I: Implications of Plasma Instabilities for the Intergalactic Magnetic Field and Extragalactic Gamma-Ray Background, Astrophys. J. 752 (2012) 22 [arXiv:1106.5494] [INSPIRE].

[57] F. Miniati and A. Elyiv, Relaxation of Blazar Induced Pair Beams in Cosmic Voids: Measurement of Magnetic Field in Voids and Thermal History of the IGM, Astrophys. J. 770 (2013) 54 [arXiv: 1208.1761] [INSPIRE].

[58] R. Durrer and A. Neronov, Cosmological Magnetic Fields: Their Generation, Evolution and Observation, Astron. Astrophys. Rev. 21 (2013) 62 [arXiv:1303.7121] [INSPIRE].

[59] M. Kawasaki, K. Kohri and N. Sugiyama, MeV scale reheating temperature and thermalization of neutrino background, Phys. Rev. D 62 (2000) 023506 [astro-ph/0002127] [INSPIRE].

[60] S. Hannestad, What is the lowest possible reheating temperature?, Phys. Rev. D 70 (2004) 043506 [astro-ph/0403291] [INSPIRE].

[61] F.L. Bezrukov and M. Shaposhnikov, The Standard Model Higgs boson as the inflaton, Phys. Lett. B 659 (2008) 703 [arXiv:0710.3755] [INSPIRE].

[62] T. Vachaspati, Magnetic fields from cosmological phase transitions, Phys. Lett. B 265 (1991) 258 [INSPIRE].

[63] J.M. Cornwall, Speculations on primordial magnetic helicity, Phys. Rev. D 56 (1997) 6146 [hep-th/9704022] [INSPIRE].

[64] T. Vachaspati, Magnetic Fields in the Aftermath of Phase Transitions, Phil. Trans. Roy. Soc. Lond. A 366 (2008) 2915 [arXiv:0802.1533] [INSPIRE].

[65] F.W. J. Olver, D.W. Lozier, R.F. Boisvert and C.W. Clark, NIST Handbook of Mathematical Functions, Cambridge University Press, 2010. 